\title{
Is it worth it? Postsecondary education and labor market outcomes for the disadvantaged
}

\author{
Ben Backes ${ }^{1}$, Harry J Holzer ${ }^{2 *}$ and Erin Dunlop Velez ${ }^{3}$
}

\author{
* Correspondence: \\ hjh4@georgetown.edu \\ ${ }^{2}$ Georgetown University and AIR/ \\ CALDER, Washington, DC 20007, \\ USA \\ Full list of author information is \\ available at the end of the article
}

\begin{abstract}
In this paper we examine a range of postsecondary education and labor market outcomes, with a particular focus on minorities and/or disadvantaged workers. We use administrative data from the state of Florida, where postsecondary student records have been linked to UI earnings data and also to secondary education records. Our main findings can be summarized as follows: 1) Gaps in secondary school achievement can account for a large portion of the variation in postsecondary attainment and labor market outcomes between the disadvantaged and other students, but meaningful gaps also exist within achievement groups, and 2) Earnings of the disadvantaged are hurt by low completion rates in postsecondary programs, poor performance during college, and not choosing high-earning fields. In particular, significant labor market premia can be earned in a variety of more technical certificate and Associate (AA) programs, even for those with weak earlier academic performance, but instead many disadvantaged (and other) students choose general humanities programs at the AA (and even the BA level) with low completion rates and low compensation afterwards. A range of policies and practices might be used to improve student choices as well as their completion rates and earnings.
\end{abstract}

JEL codes: 123, 124, J24, J31

Keywords: Disadvantaged; Postsecondary; Earnings; Achievement; Completion; Associate Degree; Bachelor's Degree

\section{Introduction}

It is, by now, well-known that rewards to college degrees (especially at the BA level) have grown quite large in the U.S. labor market. Indeed, for young people growing up in disadvantaged families, obtaining a college degree is the surest way to achieve upward mobility for themselves and their families (Isaacs 2007; Haskins et al. 2009). Thus, the incentives for low-income young people to pursue higher education have become very strong. Even if only an associate degree or a certificate is achieved, the labor market rewards for young people remain quite substantial (Holzer and Dunlop 2013).

Yet, in spite of these incentives, young economically disadvantaged students lag substantially behind their middle- and upper-income counterparts in achieving postsecondary credentials, and minorities continue to lag behind white students. While rates of college enrollment have risen for all groups in recent years, college completion rates, especially among minorities and the disadvantaged, remain low (Bound et al. 2009; Holzer and Dunlop 2013), and gaps in postsecondary attainment between income groups in the U.S. have grown in recent decades (Bailey and Dynarski 2011). In

\section{Springer}

(c) 2015 Backes et al.; licensee Springer. This is an Open Access article distributed under the terms of the Creative Commons Attribution License (http://creativecommons.org/licenses/by/4.0), which permits unrestricted use, distribution, and reproduction in any medium, provided the original work is properly credited. 
addition, while the average value of a college degree in the labor market is high, the concentration of young people in high-earning fields (like science, technology, engineering, and math, or STEM) is not as high as we might expect, especially given the meaningful differences in earnings we observe across fields. In particular, women, minorities and the poor are less likely to earn a credential in these high-earning fields.

Why are disadvantaged and/or minority students less likely than their white/middleclass counterparts to earn postsecondary credentials? In part, this is because of the academic achievement gap between these students and their more advantaged peers. This gap emerges early in life and tends to become larger as students progress through school (Reardon 2011). But even adjusting for prior achievement, disadvantaged young people lag behind others in college completion and attainment.

If the achievement gap does not fully explain this difference, what does? The research literature has identified information gaps, college costs and lack of full-time attendance as some of the factors that impair the success rates of disadvantaged young people in college (Bound et al. 2009; Hoxby and Turner 2013). Because of these factors, young disadvantaged students attend lower-quality colleges and universities, with lower completion rates, and their completion rates within the same institutions are lower than those of more advantaged students as well.

But many questions remain unanswered about the relative magnitudes and the interaction of factors that contribute to the lack of postsecondary success for disadvantaged students. For example, how important is disadvantaged students' choice of major at each level of education? Adjusting for high school achievement, by how much does their performance in college - as measured by courses taken, grades attained, credits earned, and ultimately program completion - lag behind the postsecondary performances of students who are not disadvantaged? And how much do these factors account for their lower labor market earnings, as opposed to other barriers that impede the accumulation of valuable labor market experience (Johnson and Neal, 1998)?

Answers to these questions are important if we want to design effective programs and policies to better assist disadvantaged students in their college experiences; and such answers require detailed longitudinal micro data on students, their educational institutions and experiences, and labor market outcomes. While some such information is available in existing longitudinal survey datasets on young people - such as the National Educational Longitudinal Study (NELS), High School and Beyond (HSB) or the National Longitudinal Survey of Youth (NSLY) - administrative data on students provides more detailed information on every course taken and on all academic outcomes achieved for every student who ever attended a public school in the relevant years. Until recently such data have not existed at the state level, but in several states these data are now becoming available. This enables researchers to address previously unexplored questions about the experience and outcomes associated with disadvantaged students in a number of contexts ${ }^{1}$.

In this paper, we use administrative data from the state of Florida to analyze the college and labor market experiences of two cohorts of young people. We are able to extend the current literature on the returns to college credentials in several important ways. First, the students in our data graduated high school between 2000 and 2002, making them a more recent cohort than many previous studies. Second, our large sample size, over 210,000 students, allows us to measure heterogeneous effects with precision. Third, unlike many other recent studies, we also have access to secondary school data, so we can control for earlier 
achievement. Finally, this paper focuses on disadvantaged students in particular, a large and growing fraction of the U.S. postsecondary market.

Below, we describe our data and analysis in Part II of the paper, present the empirical results in Part III, and present our conclusions and their general implications for further research and policy in Part IV.

\section{Data and analysis plan}

\subsection{Data overview}

To create our sample, we merge together three large administrative student-level data sets from the state of Florida: secondary school data, postsecondary data, and unemployment earnings data, the latter of which provides earnings information for nearly all Florida residents ${ }^{2}$. Linked together by a unique individual identifier, we are able to follow students from eighth grade through college (and graduate school) and their entry into the labor force, provided the student does not leave the state of Florida. Our data provide large sample sizes unavailable in national surveys, offer rich information to account for selection based on ability (e.g. Lovenheim and Reynolds 2011), and provide detailed information on several important outcome measures.

The data for this paper follow two cohorts of students. The first wave comprises all students who began 10th grade at a Florida public school in 1997-98. The second wave consists of all students who began 8th grade in 1997-1998. The data set contains observations as recent as 2011-12, so we observe 10-12 years of postsecondary and labor market outcomes.

The secondary student-level data include student demographic information (race, gender, and limited English proficiency status), and we use eligibility for free- or reducedprice lunch (FRL) as a measure of family income. In addition, we have data on courses taken, course grades, GPA, and standardized test scores (such as the Florida Comprehensive Assessment Test, or FCAT). These serve as important controls and allow for analysis of heterogeneous treatment effects.

Our rich postsecondary information comes from public colleges and universities in Florida and includes date and institution of each enrollment instance, courses taken at each institution and grades received, cumulative credits earned, field of study, and degree attainment. We utilize this data to measure both intermediate postsecondary outcomes (such as major choice) and terminal postsecondary outcomes (such as highest degree earned).

Finally, we merge our data with quarterly wage information from employer reports to Florida's Unemployment Insurance (UI) office. These data are collected during high school and for five years after students leave their last educational institution.

The main drawback of our data is their limitation to a single state, which has implications for both generalizability to other states and attrition (since individuals leaving the state are not observed). For generalizability, this drawback is somewhat mitigated by Florida's large and diverse makeup. According to the 2012 Digest of Education Statistics, Florida ranked as the fourth largest state in terms of the number of graduates from public high schools. In addition, 22 percent of its high school graduates were black and 23 percent Hispanic, compared to national averages of 15 and 16 percent, respectively. Given this paper's focus on disadvantaged students, using data from such a large and highly diverse state increases sample sizes and the precision of our estimates for these groups. 
Although the data are limited to students who attend in-state public institutions, Florida has a relatively low out-migration rate for college-going. Specifically, only 10 percent of Floridian first-time degree or certificate-seeking undergraduates attend college out of state $^{3}$. In addition, about $85 \%$ percent of first-time degree-seeking students attend a public, rather than private, institution in Florida ${ }^{4}$. Therefore, we likely do not lose a large subset of our sample to private or out-of-state colleges. However, students who enroll in out-of-state or private colleges are more likely to come from higher-income families, are higher-performing students, and may be attending higherquality institutions. The potential downward bias in some of our estimates of enrollment, completion, and earnings due to missing student observations needs to be kept in mind as we review our results.

In addition, as many as half of all Florida higher education enrollees do not subsequently appear in the labor market data. This could be either because they have moved out of state for work, cannot find employment, or have chosen not to enter the labor market. The first of these explanations might generate some under-representation of high-earning groups in our data, while the others might instead generate some underrepresentation of low earners. Previous studies have found that $30-50 \%$ of males age 25-45 work in a state other than their birth state, depending on their level of education, which is broadly consistent with the out-migration we observe 5 . In addition, our own calculations using the American Community Survey find similar rates of mobility of college graduates who were born in Florida. Overall, rates of labor market participation appear lowest for those with the weakest observed skills in their high school years ${ }^{6}$.

A final limitation of the sample is its emphasis on traditional students: those whose postsecondary studies begin relatively soon after high school graduation. For example, if a student were to go back to college outside the 10-12 year window for which we have data, this enrollment would not be captured. Thus, we miss out on this important component of the community college-going population ${ }^{7}$.

We construct several variables from our administrative data. For example, for students who complete a degree, identifying degree earned and field of study is straightforward. However, students who enroll in postsecondary education but do not complete a degree could have had many fields of study throughout the course of their enrollment. Reasonable choices for "field of study" include first declared major, last declared major, or most frequently-observed major (by number of terms). In this paper, for those students who do not earn a degree, we consider their field of study to be their final declared major before exiting postsecondary schooling.

Finally, we count each quarter that an individual appears in the UI data as one quarter of work experience. When calculating tenure, we count each quarter of employment with a given employer, whether or not that employment is continuous ${ }^{8}$. When examining labor market outcomes, we only consider observations following each individual's final term of postsecondary enrollment and only include students 18 years of age and older. Only those quarters with positive earnings are included in our computations ${ }^{9}$.

\subsection{Analysis plan}

A number of recent studies have examined labor market returns to various fields and credentials (Jepsen et al. 2014 (Kentucky); Bahr et al. 2014 (Michigan); Bahr et al. 2014 (California); Belfield et al. 2014 (North Carolina)). However, the data underlying these 
studies lack information about students' high school experiences. In order to estimate postsecondary returns, these studies compare pre-schooling earnings with postschooling earnings in a fixed effect framework and attribute the post-schooling earnings gain to the effect of postsecondary training. However, a recent study by $\mathrm{Xu}$ and Jaggers (2014) provides evidence that pre-schooling wages of younger students understate their actual earning potential and that the assumptions underlying fixed effects estimation may not hold. Therefore, we control for a student's academic performance in high school as a measure of their earning potential.

We begin our analysis by presenting summary data on differences in higher education and labor market outcomes between race/gender groups, and those who do and do not qualify for FRL. After establishing the basic facts on outcomes that need to be explained, we divide the students into quartiles based on their high school FCAT scores and explore differences between these quartiles - which likely reflect their differences in early achievement - versus those differences within quartiles, which cannot be attributed to achievement gaps ${ }^{10}$.

We then present results from regression equations of the following general form:

$\operatorname{lnEARN} N_{i k t}=f\left(E D_{i}, X_{i}\right.$, ACHIEVE $_{i}$, EXP $\left._{\mathrm{i}}, \mathrm{EXP}_{\mathrm{i}}{ }^{2}, \mathrm{TEN}_{\mathrm{i}}, \mathrm{TEN}_{\mathrm{i}}{ }^{2}, \mathrm{COHORT}_{\mathrm{k}}, \mathrm{TIME}_{\mathrm{t}}\right)+\mathrm{u}_{\mathrm{ikt}}$

where lnEARN denotes the natural log of quarterly earnings; ED denotes the highest level of education completed (either high school, a certificate, an AA degree, a BA degree, or higher); X measures race/ethnicity, gender, and family background; ${ }^{11}$ ACHIEVE measures high school achievement (FCAT math score); ${ }^{12}$ EXP and TEN reflect quarters of total labor market experience and job tenure with their current firm respectively (entering the equation in both linear and quadratic form); and COHORT and TIME denote cohort and year/quarter dummies. The individual person, cohort, and year/quarter are denoted respectively by the subscripts $i, k$, and $t$. Missing values in achievement measures are measured as zeroes along with a "1" for a missing value dummy variable.

We use our measure of high school achievement to correct for selection into higher education, which, as discussed above, is unobservable in most studies. For the reasons noted above, we regard fixed effects models as not appropriate in this setting and thus do not estimate them.

In some versions of the estimated equations, we control for the number of postsecondary credits earned if the student did not complete the degree program in which they had enrolled. In other equations, we add measures of postsecondary achievement - such as college GPA and the number of credits earned in math or science courses - to see the extent to which such achievement is rewarded in the job market. Returns to different fields of study at different levels of higher education are estimated in separate equations as well. Finally, we separate out those achieving different types of associate degrees (such as Associate in Arts v. Associate in Applied Science, or AA v. AAS) to see the extent to which the kind of degree achieved affects subsequent labor market earnings as well.

\section{Empirical results}

\subsection{Summary results}

Table 1 presents our estimates of higher education and labor market outcomes for Florida students in our sample. These outcomes include quarterly earnings as well as 
Table 1 Education and labor market outcomes: summary statistics

\begin{tabular}{ll}
\hline A. All students and workers \\
\hline Quarterly earnings & $\$ 5,227.36$ \\
& $(4572.22)$ \\
N & $3,975,013$ \\
Educational attainment & \\
HS & 0.693 \\
Voc/Cert & 0.044 \\
AA & 0.159 \\
BA or above & 0.193 \\
N & 393,213 \\
Enrollment & \\
Voc/Cert & 0.106 \\
AA & 0.529 \\
BA & 0.283 \\
Completion & \\
Voc/Cert & 0.419 \\
AA & 0.300 \\
BA & 0.592 \\
\hline B B race/gender
\end{tabular}

B. By race/gender

\begin{tabular}{|c|c|c|c|c|c|c|}
\hline & \multicolumn{2}{|l|}{ White } & \multicolumn{2}{|l|}{ Black } & \multicolumn{2}{|l|}{ Hispanic } \\
\hline & Male & Female & Male & Female & Male & Female \\
\hline \multirow[t]{2}{*}{ Quarterly earnings } & $\$ 5,746.43$ & $5,245.16$ & $4,237.60$ & $4,140.88$ & $5,654.21$ & $5,473.62$ \\
\hline & $(4967.67)$ & $(4575.25)$ & (3779.69) & (3625.38) & $(4638.84)$ & $(4332.01)$ \\
\hline $\mathrm{N}$ & $1,243,071$ & 906,996 & 528,548 & 463,994 & 415,221 & 320,809 \\
\hline
\end{tabular}

Educational attainment

$\begin{array}{lllllll}\text { HS } & 0.716 & 0.774 & 0.531 & 0.664 & 0.619 & 0.700 \\ \text { Voc/Cert } & 0.063 & 0.037 & 0.034 & 0.040 & 0.048 & 0.025 \\ \text { AA } & 0.137 & 0.209 & 0.066 & 0.132 & 0.135 & 0.206 \\ \text { BA or above } & 0.168 & 0.266 & 0.075 & 0.170 & 0.125 & 0.217\end{array}$

Enrollment

$\begin{array}{lllllll}\text { Voc/Cert } & 0.113 & 0.082 & 0.121 & 0.147 & 0.116 & 0.085 \\ \text { AA } & 0.459 & 0.561 & 0.433 & 0.594 & 0.556 & 0.640 \\ \text { BA } & 0.255 & 0.347 & 0.157 & 0.265 & 0.224 & 0.322\end{array}$

Completion

\begin{tabular}{lllllll} 
Voc/Cert & 0.555 & 0.445 & 0.281 & 0.274 & 0.414 & 0.289 \\
AA & 0.299 & 0.373 & 0.153 & 0.222 & 0.243 & 0.322 \\
BA & 0.580 & 0.658 & 0.422 & 0.549 & 0.494 & 0.573 \\
\hline
\end{tabular}

C. By FRL/gender

\begin{tabular}{|c|c|c|c|c|}
\hline & \multicolumn{2}{|l|}{ Non-FRL } & \multicolumn{2}{|l|}{ FRL } \\
\hline & Male & Female & Male & Female \\
\hline \multirow[t]{2}{*}{ Quarterly earnings } & $\$ 5,943.05$ & $5,610.97$ & $4,740.15$ & $4,236.17$ \\
\hline & $(5138.77)$ & (4755.39) & (3965.59) & (3555.97) \\
\hline $\mathrm{N}$ & $1,241,573$ & 968,371 & 865,102 & 698,666 \\
\hline
\end{tabular}


Table 1 Education and labor market outcomes: summary statistics (Continued)

\begin{tabular}{lllll}
\hline Educational attainment & & & & \\
HS & 0.744 & 0.812 & 0.589 & 0.671 \\
Voc/Cert & 0.059 & 0.035 & 0.044 & 0.037 \\
AA & 0.145 & 0.217 & 0.087 & 0.147 \\
BA or above & 0.187 & 0.295 & 0.065 & 0.126 \\
Enrollment & & & & \\
Voc/Cert & 0.113 & 0.085 & 0.118 & 0.120 \\
AA & 0.501 & 0.590 & 0.448 & 0.588 \\
BA & 0.288 & 0.389 & 0.139 & 0.211 \\
Completion & & & & \\
Voc/Cert & 0.521 & 0.408 & 0.377 & 0.310 \\
AA & 0.290 & 0.368 & 0.194 & 0.249 \\
BA & 0.572 & 0.648 & 0.421 & 0.519 \\
\hline
\end{tabular}

Notes: The number of quarterly earning observations are the number of labor market quarters observed while educational outcomes are calculated out of unique students. Standard deviations for continuous variables are in parentheses below their corresponding means. All postsecondary attainment and enrollment are conditional upon HS graduation. Completion for a degree level is conditional upon enrollment in that degree.

observed high school completion rates; higher educational attainment and enrollment rates, conditional on completing high school; and higher education completion rates, conditional on enrollment. These results appear for all students and workers in part A, and then separately for race/gender groups and for FRL/gender groups respectively in parts B and C.

The results in part A show that quarterly earnings for all workers in our sample average about $\$ 5,200$ and have considerable variation, though this estimate is likely biased by out-of-state migration of higher-achieving students and workers on the high end of the wage distribution, and by labor force nonparticipation on the low end ${ }^{13}$. Just over two-thirds of all students complete high school - a rate roughly consistent with computations using administrative data from other states in this period ${ }^{14}$.

In our sample, 11,53 , and $28 \%$ of high school graduates enroll in vocational certificate, AA, and BA programs, respectively. As shown in part B, both Hispanic males and females are relatively more likely to enroll in AA programs, with Hispanic females being the most likely to pursue an AA degree (64\%) and black males being the least likely (43\%). On the other hand, white students are relatively more likely to enroll in a BA program.

The data also indicate that 4,16, and 19\% of high school graduates obtain vocational certificates, AA, and BA degrees, respectively, in public institutions in Florida. These numbers are substantially lower than the share of students who enroll, due to low completion rates, especially for those enrolled in AA programs. Large differences in completion rates are apparent across racial/gender groups. For every 100 white male students who enroll in an AA program, about 30 will earn an associate's degree, compared to 15 for black males and 24 for Hispanic males. As shown in Table 1C, completion rates are similarly lower for FRL students relative to non-FRL students.

It should be noted that the BA enrollment and completion rates are also likely downward biased by the out-migration of higher-achieving and higher-income students in these data, as noted above, but the observed AA rates are likely much less downward-biased by these factors. The fact that completion rates are somewhat higher 
in vocational certificate programs than in AA programs, though overall enrollments are much lower, is notable as well.

Overall in Table 1, we find numerous results, many of which have been observed elsewhere (e.g., Holzer and Dunlop, op. cit):

- Whites earn more than blacks and have higher rates of educational attainment, enrollment and completion than blacks or Hispanics;

- The poor, (as measured by FRL) also have lower rates of attainment, enrollment, and completion; and

- Educational outcomes are generally higher for females in each racial or income group though their earnings are lower.

We also note that educational attainment and labor market outcomes for Hispanics are consistently better than those for blacks; this partly, but not entirely, reflects the presence of higher-achieving and higher-earning Cubans among the latter (Borjas 1987). However, outcomes tend to be better for non-Cuban Hispanics too ${ }^{15}$. Higher education and labor market outcomes of those from FRL households are generally similar to, or a bit better than, those observed for blacks in Florida.

Two other outcomes are notable as well: first, males are generally more likely than females to enroll in and complete vocational programs; and second, black males lag behind black females in enrollment and completion by even more than those of other racial groups, and they earn only marginally more than the females in the job market. For instance, the rate of both BA and AA attainment for black females is more than twice as high as that of black males. While $17 \%$ of black females earn a bachelor's degree, less than $8 \%$ of black males do. It is also important to note that the general underrepresentation of black (and/or) low-income men that we commonly find in survey data might create less bias in these administrative data, but perhaps some bias still exists ${ }^{16}$.

Table 2 presents some additional estimates of intermediate higher education and labor market outcomes. These include our measures of high school achievement GPA and math FCAT - as well as somewhat similar measures of postsecondary achievement - postsecondary GPA, credits earned (among all students, whether or not they have completed their programs), and numbers of math or science credits. Intermediate outcomes for the labor market - work experience and job tenure - appear as well. Again, estimates of outcomes appear for the entire sample of students and workers in part A, and for race/gender and FRL/gender groups in parts $\mathrm{B}$ and $\mathrm{C}$ of the table, respectively.

The results in part A show high school and postsecondary GPAs that average about 2.2. About a fourth of all postsecondary credits earned are in math or science, and most workers have accumulated over 4 years of work experience on average over our sample period, with a third of these quarters being with their current/most recent employer.

Comparing these outcomes across race/gender and FRL/gender groups generates findings similar to those in Table 1 . Namely, males generally have more labor market experience or tenure than females in each group (with black students being the exception) but lower GPAs and postsecondary credits earned. Results for FCAT math scores are more mixed - with higher scores among males than females in most groups but lower 
Table 2 Intermediate education and labor market outcomes: summary statistics

\begin{tabular}{|c|c|c|c|c|c|c|}
\hline \multicolumn{7}{|l|}{ A. All students/workers } \\
\hline \multicolumn{7}{|l|}{ Intermediate HS outcomes } \\
\hline \multirow[t]{2}{*}{ 10th grade FCAT math score } & 0.000 & & & & & \\
\hline & $(1.000)$ & & & & & \\
\hline \multirow[t]{2}{*}{ HS GPA } & 2.208 & & & & & \\
\hline & $(0.982)$ & & & & & \\
\hline \multicolumn{7}{|c|}{ Intermediate postsecondary outcomes } \\
\hline \multirow[t]{2}{*}{ Postsecondary GPA } & 2.271 & & & & & \\
\hline & $(1.083)$ & & & & & \\
\hline \multirow[t]{2}{*}{ Total postsecondary credits } & 71.450 & & & & & \\
\hline & $(61.321)$ & & & & & \\
\hline \multirow[t]{2}{*}{ Postsecondary math credits } & 7.730 & & & & & \\
\hline & $(8.207)$ & & & & & \\
\hline \multirow[t]{2}{*}{ Postsecondary science credits } & 11.016 & & & & & \\
\hline & $(17.469)$ & & & & & \\
\hline \multicolumn{7}{|l|}{ Labor market inputs } \\
\hline \multirow[t]{2}{*}{ Work experience } & 18.886 & & & & & \\
\hline & $(11.272)$ & & & & & \\
\hline \multirow[t]{2}{*}{ Tenure } & 6.206 & & & & & \\
\hline & $(6.897)$ & & & & & \\
\hline \multicolumn{7}{|l|}{ B. By race/gender } \\
\hline & \multicolumn{2}{|l|}{ White } & \multicolumn{2}{|l|}{ Black } & \multicolumn{2}{|l|}{ Hispanic } \\
\hline & Male & Female & Male & Female & Male & Female \\
\hline \multicolumn{7}{|l|}{ Intermediate HS outcomes } \\
\hline \multirow[t]{2}{*}{ 10th grade FCAT math score } & 0.287 & 0.264 & -0.691 & -0.561 & -0.149 & -0.175 \\
\hline & $(0.922)$ & $(0.822)$ & $(1.074)$ & $(0.966)$ & $(1.006)$ & $(0.913)$ \\
\hline \multirow[t]{2}{*}{ HS GPA } & 2.188 & 2.533 & 1.804 & 2.087 & 1.945 & 2.263 \\
\hline & $(0.994)$ & $(0.972)$ & $(0.891)$ & $(0.912)$ & $(0.920)$ & $(0.912)$ \\
\hline \multicolumn{7}{|c|}{ Intermediate postsecondary outcomes } \\
\hline \multirow[t]{2}{*}{ Postsecondary GPA } & 2.266 & 2.568 & 1.718 & 1.967 & 2.062 & 2.301 \\
\hline & $(1.102)$ & $(0.995)$ & $(1.103)$ & $(1.048)$ & $(1.089)$ & $(1.013)$ \\
\hline \multirow[t]{2}{*}{ Total postsecondary credits } & 68.515 & 79.069 & 50.597 & 68.157 & 63.976 & 77.191 \\
\hline & $(60.08)$ & $(60.723)$ & $(57.608)$ & $(63.531)$ & $(58.351)$ & $(60.534)$ \\
\hline \multirow[t]{2}{*}{ Postsecondary math credits } & 7.657 & 7.732 & 6.055 & 7.347 & 8.261 & 8.800 \\
\hline & $(8.682)$ & $(7.189)$ & $(8.573)$ & $(8.062)$ & (9.153) & $(8.104)$ \\
\hline \multirow[t]{2}{*}{ Postsecondary science credits } & 9.331 & 12.999 & 6.53 & 11.4 & 8.518 & 12.219 \\
\hline & $(15.527)$ & $(18.504)$ & $(13.53)$ & $(18.211)$ & $(14.853)$ & (18.246) \\
\hline \multicolumn{7}{|l|}{ Labor market inputs } \\
\hline Work experience & 19.451 & 19.12 & 17.703 & 18.401 & 19.027 & 18.955 \\
\hline & $(11.397)$ & $(10.881)$ & $(11.567)$ & $(11.214)$ & (11.536) & (11.109) \\
\hline Tenure & 6.500 & 6.357 & 5.616 & 5.723 & 6.174 & 6.421 \\
\hline & $(7.274)$ & $(6.794)$ & $(6.696)$ & $(6.437)$ & $(6.882)$ & $(6.747)$ \\
\hline
\end{tabular}


Table 2 Intermediate education and labor market outcomes: summary statistics (Continued)

\begin{tabular}{|c|c|c|c|c|}
\hline \multicolumn{5}{|l|}{ C. By FRL/gender } \\
\hline & \multicolumn{2}{|l|}{ Non-FRL } & \multicolumn{2}{|l|}{ FRL } \\
\hline & Male & Female & Male & Female \\
\hline \multicolumn{5}{|l|}{ Intermediate HS outcomes } \\
\hline \multirow[t]{2}{*}{ 10th grade FCAT math score } & 0.242 & 0.220 & -0.381 & -0.398 \\
\hline & $(0.954)$ & $(0.856)$ & $(1.035)$ & $(0.928)$ \\
\hline \multirow[t]{2}{*}{ HS GPA } & 2.286 & 2.628 & 1.805 & 2.088 \\
\hline & $(0.936)$ & $(0.901)$ & $(0.909)$ & $(0.931)$ \\
\hline \multicolumn{5}{|c|}{ Intermediate postsecondary outcomes } \\
\hline \multirow[t]{2}{*}{ Postsecondary GPA } & 2.240 & 2.531 & 1.877 & 2.072 \\
\hline & $(1.075)$ & $(0.970)$ & $(1.153)$ & $(1.090)$ \\
\hline \multirow[t]{2}{*}{ Total postsecondary credits } & 71.219 & 83.558 & 50.689 & 62.575 \\
\hline & $(61.049)$ & $(61.769)$ & $(54.998)$ & $(59.091)$ \\
\hline \multirow[t]{2}{*}{ Postsecondary math credits } & 8.097 & 8.253 & 6.496 & 7.266 \\
\hline & (8.926) & $(7.509)$ & $(8.692)$ & $(7.932)$ \\
\hline \multirow[t]{2}{*}{ Postsecondary science credits } & 9.927 & 14.052 & 6.620 & 10.037 \\
\hline & $(16.161)$ & $(19.571)$ & (13.549) & (16.577) \\
\hline \multicolumn{5}{|l|}{ Labor market inputs } \\
\hline \multirow[t]{2}{*}{ Work experience } & 19.388 & 19.381 & 18.432 & 18.34 \\
\hline & $(11.475)$ & $(10.941)$ & $(11.441)$ & (11.089) \\
\hline \multirow[t]{2}{*}{ Tenure } & 6.536 & 6.438 & 5.757 & 5.843 \\
\hline & $(7.256)$ & $(6.782)$ & $(6.661)$ & $(6.509)$ \\
\hline
\end{tabular}

Note: Intermediate HS and postsecondary outcomes are calculated out of numbers of unique students, while labor market inputs are calculated out of labor market quarters.

scores among black males than females. Once again, minorities score lower than whites on most academic measures and FRL students score lower than non-FRL. The average white male accumulates about 68 postsecondary credits, compared to 51 for black males and 64 for Hispanic males. In addition, Hispanics outperform blacks (and FRL) on academic measures, and even accumulate nearly as much work experience as whites. Black males lag behind their female counterparts in educational outcomes by more than we observe for other groups. For example, the average black female earns 68 postsecondary credits with a postsecondary GPA of about 2.0, while the average black male earns 51 credits with an average GPA of 1.7 .

\subsection{Selection by high school achievement}

Undoubtedly, young people with different levels of academic achievement self-select into different higher education programs, institutions, and fields of study and differ in terms of their labor market participation and occupational choices. We investigate the extent to which differences in academic or labor market performance reflect differential selection based on students' ability and early academic achievement.

Table 3 presents the full range of higher education and labor market outcomes observed in earlier tables, but this time, they appear separately for individuals who fall into each of the four quartiles (where 1 is lowest and 4 is highest) on the high school math FCAT test. Parts A, B, and C of these tables reflect summary outcomes for all 
Table 3 Education and labor market outcomes: by hs achievement quartile

A. All students/workers

\begin{tabular}{|c|c|c|c|c|}
\hline & Quartile 1 & Quartile 2 & Quartile 3 & Quartile 4 \\
\hline \multirow[t]{2}{*}{ Quarterly earnings } & $\$ 4,383.75$ & $5,227.19$ & $6,159.69$ & $8,072.06$ \\
\hline & (3411.79) & $(3955.62)$ & $(4765.38)$ & $(6445.16)$ \\
\hline \multicolumn{5}{|l|}{ Educational attainment } \\
\hline Voc/Cert & 0.045 & 0.054 & 0.054 & 0.035 \\
\hline AA & 0.082 & 0.154 & 0.220 & 0.224 \\
\hline BA or above & 0.041 & 0.104 & 0.226 & 0.453 \\
\hline \multicolumn{5}{|l|}{ Enrollment } \\
\hline Voc/Cert & 0.143 & 0.119 & 0.100 & 0.060 \\
\hline AA & 0.536 & 0.590 & 0.600 & 0.484 \\
\hline BA & 0.089 & 0.190 & 0.351 & 0.580 \\
\hline \multicolumn{5}{|l|}{ Completion } \\
\hline Voc/Cert & 0.317 & 0.456 & 0.542 & 0.585 \\
\hline AA & 0.153 & 0.261 & 0.367 & 0.462 \\
\hline BA & 0.425 & 0.500 & 0.577 & 0.656 \\
\hline \multicolumn{5}{|l|}{ Intermediate HS outcomes } \\
\hline \multirow[t]{2}{*}{ 10th grade FCAT Math Score } & -1.286 & -0.194 & 0.368 & 1.131 \\
\hline & $(0.823)$ & $(0.170)$ & $(0.166)$ & $(0.404)$ \\
\hline \multirow[t]{2}{*}{ HS GPA } & 1.977 & 2.342 & 2.680 & 3.159 \\
\hline & $(0.679)$ & $(0.647)$ & $(0.640)$ & $(0.588)$ \\
\hline
\end{tabular}


Table 3 Education and labor market outcomes: by hs achievement quartile (Continued)

\begin{tabular}{lllll}
\hline $\begin{array}{l}\text { Intermediate postsecondary } \\
\text { outcomes }\end{array}$ & & & & \\
Postsecondary GPA & 1.734 & 2.120 & 2.427 & 2.841 \\
& $(1.085)$ & $(0.995)$ & $(0.930)$ & $(0.810)$ \\
Total postsecondary credits & 43.542 & 62.603 & 80.698 & 101.912 \\
& $(49.200)$ & $(56.430)$ & $(59.703)$ & $(61.394)$ \\
Postsecondary math credits & 5.401 & 7.957 & 8.893 & 9.481 \\
& $(7.706)$ & $(8.079)$ & $(7.736)$ & $(8.558)$ \\
Postsecondary science credits & 5.476 & 8.908 & 12.638 & 17.412 \\
& $(10.805)$ & $(14.077)$ & $(17.949)$ & $(22.212)$
\end{tabular}

\section{Labor market inputs}

\begin{tabular}{|c|c|c|c|c|}
\hline \multirow[t]{2}{*}{ Work experience } & 18.997 & 19.323 & 19.486 & 18.843 \\
\hline & $(11.453)$ & $(11.063)$ & (10.758) & (10.032) \\
\hline \multirow[t]{2}{*}{ Tenure } & 6.187 & 6.619 & 6.778 & 6.831 \\
\hline & $(6.944)$ & $(7.126)$ & $(7.002)$ & (6.669) \\
\hline
\end{tabular}

\begin{tabular}{|c|c|c|c|c|c|c|c|c|c|c|c|c|}
\hline \multicolumn{13}{|l|}{ B. By race } \\
\hline & \multicolumn{3}{|c|}{ Quartile 1} & \multicolumn{3}{|c|}{ Quartile 2} & \multicolumn{3}{|c|}{ Quartile 3} & \multicolumn{3}{|c|}{ Quartile 4} \\
\hline & White & Black & Hispanic & White & Black & $\overline{\text { Hispanic }}$ & White & Black & $\overline{\text { Hispanic }}$ & White & Black & $\overline{\text { Hispanic }}$ \\
\hline \multirow[t]{2}{*}{ Quarterly earnings } & $\$ 4,455.57$ & $4,036.84$ & $4,987.81$ & $5,190.27$ & $4,823.83$ & $5,791.99$ & $6,085.60$ & $5,687.52$ & $6,711.49$ & $7,934.70$ & $7,793.08$ & $8,626.83$ \\
\hline & (3528.71) & (3187.7) & (3553.55) & (3967.44) & (3684.96) & (4138.44) & (4703.92) & $(4516.54)$ & (4982.39) & (6312.65) & (6599.3) & $(6615.71)$ \\
\hline
\end{tabular}

Educational attainment

\begin{tabular}{|c|c|c|c|c|c|c|c|c|c|c|c|c|}
\hline Voc/Cert & 0.058 & 0.041 & 0.032 & 0.063 & 0.047 & 0.042 & 0.061 & 0.038 & 0.044 & 0.038 & 0.020 & 0.029 \\
\hline $\mathrm{AA}$ & 0.069 & 0.075 & 0.118 & 0.149 & 0.133 & 0.186 & 0.226 & 0.162 & 0.237 & 0.232 & 0.166 & 0.211 \\
\hline BA & 0.030 & 0.045 & 0.049 & 0.084 & 0.133 & 0.109 & 0.207 & 0.280 & 0.240 & 0.446 & 0.470 & 0.444 \\
\hline
\end{tabular}


Table 3 Education and labor market outcomes: by hs achievement quartile (Continued)

\begin{tabular}{|c|c|c|c|c|c|c|c|c|c|c|c|c|}
\hline \multicolumn{13}{|l|}{ Enrollment } \\
\hline Voc/Cert & 0.135 & 0.159 & 0.120 & 0.127 & 0.118 & 0.102 & 0.105 & 0.093 & 0.089 & 0.063 & 0.047 & 0.054 \\
\hline AA & 0.442 & 0.559 & 0.629 & 0.556 & 0.582 & 0.674 & 0.598 & 0.542 & 0.644 & 0.494 & 0.413 & 0.481 \\
\hline BA & 0.060 & 0.100 & 0.111 & 0.148 & 0.249 & 0.210 & 0.314 & 0.444 & 0.386 & 0.566 & 0.637 & 0.600 \\
\hline \multicolumn{13}{|l|}{ Completion } \\
\hline Voc/Cert & 0.432 & 0.260 & 0.268 & 0.499 & 0.398 & 0.411 & 0.578 & 0.410 & 0.499 & 0.603 & 0.423 & 0.535 \\
\hline AA & 0.155 & 0.134 & 0.188 & 0.267 & 0.228 & 0.276 & 0.378 & 0.298 & 0.368 & 0.469 & 0.402 & 0.439 \\
\hline BA & 0.474 & 0.414 & 0.394 & 0.539 & 0.478 & 0.464 & 0.595 & 0.550 & 0.549 & 0.665 & 0.590 & 0.622 \\
\hline \multicolumn{13}{|l|}{ Intermediate HS outcomes } \\
\hline \multirow[t]{2}{*}{ 10th grade FCAT Math Score } & -1.175 & -1.385 & -1.260 & -0.181 & -0.217 & -0.199 & 0.377 & 0.333 & 0.358 & 1.141 & 1.015 & 1.081 \\
\hline & $(0.762)$ & $(0.869)$ & $(0.796)$ & $(0.169)$ & $(0.17)$ & $(0.171)$ & $(0.165)$ & $(0.163)$ & $(0.165)$ & $(0.409)$ & $(0.305)$ & $(0.349)$ \\
\hline \multirow[t]{2}{*}{ HS GPA } & 1.998 & 1.962 & 1.968 & 2.340 & 2.364 & 2.308 & 2.688 & 2.696 & 2.616 & 3.176 & 3.046 & 3.052 \\
\hline & $(0.722)$ & $(0.653)$ & $(0.668)$ & $(0.665)$ & $(0.617)$ & $(0.637)$ & $(0.654)$ & $(0.601)$ & $(0.618)$ & $(0.594)$ & $(0.550)$ & $(0.573)$ \\
\hline \multicolumn{13}{|l|}{$\begin{array}{l}\text { Intermediate postsecondary } \\
\text { outcomes }\end{array}$} \\
\hline \multirow[t]{2}{*}{ Postsecondary GPA } & 1.849 & 1.646 & 1.767 & 2.179 & 1.999 & 2.120 & 2.463 & 2.261 & 2.423 & 2.857 & 2.653 & 2.788 \\
\hline & $(1.142)$ & $(1.047)$ & $(1.06)$ & $(1.029)$ & $(0.936)$ & $(0.981)$ & $(0.941)$ & $(0.890)$ & $(0.911)$ & $(0.816)$ & $(0.786)$ & $(0.792)$ \\
\hline \multirow[t]{2}{*}{ Total postsecondary credits } & 38.880 & 44.080 & 49.196 & 56.596 & 69.716 & 65.880 & 76.475 & 91.804 & 84.474 & 100.025 & 114.108 & 102.806 \\
\hline & (44.219) & $(50.849)$ & $(51.569)$ & (52.473) & $(61.426)$ & $(56.218)$ & $(57.904)$ & $(65.152)$ & $(59.144)$ & $(60.809)$ & $(66.257)$ & $(59.791)$ \\
\hline \multirow[t]{2}{*}{ Postsecondary math credits } & 4.701 & 5.302 & 6.586 & 7.144 & 8.458 & 9.052 & 8.312 & 9.727 & 10.021 & 9.149 & 10.817 & 10.337 \\
\hline & $(6.97)$ & $(7.739)$ & $(8.468)$ & $(7.556)$ & $(8.321)$ & $(8.657)$ & $(7.341)$ & $(8.491)$ & $(8.068)$ & $(8.337)$ & $(9.398)$ & $(8.614)$ \\
\hline \multirow[t]{2}{*}{ Postsecondary science credits } & 4.585 & 5.752 & 5.992 & 7.692 & 10.674 & 8.908 & 11.485 & 15.867 & 12.958 & 16.469 & 21.914 & 18.003 \\
\hline & $(9.041)$ & $(11.297)$ & $(11.547)$ & $(12.14)$ & $(16.431)$ & $(13.651)$ & $(16.444)$ & (20.978) & $(18.201)$ & $(21.238)$ & $(26.452)$ & $(22.569)$ \\
\hline
\end{tabular}


Table 3 Education and labor market outcomes: by hs achievement quartile (Continued)

\begin{tabular}{|c|c|c|c|c|c|c|c|c|c|c|c|c|}
\hline \multicolumn{13}{|l|}{ Labor market inputs } \\
\hline \multirow[t]{2}{*}{ Work experience } & 19.681 & 18.525 & 18.973 & 19.707 & 18.560 & 19.439 & 19.804 & 18.407 & 19.277 & 19.163 & 17.461 & 18.082 \\
\hline & $(11.565)$ & $(11.373)$ & $(11.43)$ & (11.138) & $(10.843)$ & (11.129) & $(10.826)$ & $(10.495)$ & $(10.672)$ & $(10.084)$ & $(9.767)$ & (9.833) \\
\hline \multirow[t]{2}{*}{ Tenure } & 6.417 & 5.968 & 6.286 & 6.867 & 6.061 & 6.692 & 6.970 & 5.996 & 6.672 & 6.952 & 6.044 & 6.550 \\
\hline & $(7.292)$ & $(6.736)$ & $(6.793)$ & $(7.445)$ & $(6.529)$ & $(6.989)$ & $(7.212)$ & $(6.312)$ & $(6.679)$ & $(6.797)$ & $(5.927)$ & $(6.257)$ \\
\hline \multicolumn{13}{|l|}{ C. By FRL status } \\
\hline & \multicolumn{2}{|c|}{ Quartile 1} & \multicolumn{2}{|c|}{ Quartile 2} & \multicolumn{2}{|c|}{ Quartile 3} & \multicolumn{2}{|l|}{ Quartile 4} & & & & \\
\hline & Non-FRL & FRL & Non-FRL & FRL & Non-FRL & FRL & Non-FRL & FRL & & & & \\
\hline \multirow[t]{2}{*}{ Quarterly earnings } & $\$ 4,644.73$ & $4,220.75$ & $5,487.59$ & $4,882.10$ & $6,438.89$ & $5,545.63$ & $8,350.22$ & $6,737.48$ & & & & \\
\hline & $(3608.34)$ & (3262.46) & $(4145.20)$ & $(3664.24)$ & $(4917.97)$ & (4359.39) & $(6593.75)$ & $(5459.76)$ & & & & \\
\hline \multicolumn{13}{|c|}{ Educational attainment } \\
\hline Voc/Cert & 0.054 & 0.039 & 0.059 & 0.046 & 0.057 & 0.047 & 0.034 & 0.039 & & & & \\
\hline $\mathrm{AA}$ & 0.092 & 0.075 & 0.172 & 0.126 & 0.237 & 0.175 & 0.226 & 0.212 & & & & \\
\hline BA & 0.049 & 0.034 & 0.120 & 0.078 & 0.249 & 0.162 & 0.480 & 0.300 & & & & \\
\hline \multicolumn{13}{|l|}{ Enrollment } \\
\hline Voc/Cert & 0.144 & 0.144 & 0.122 & 0.113 & 0.102 & 0.095 & 0.057 & 0.073 & & & & \\
\hline $\mathrm{AA}$ & 0.552 & 0.536 & 0.610 & 0.556 & 0.614 & 0.559 & 0.479 & 0.521 & & & & \\
\hline BA & 0.100 & 0.082 & 0.209 & 0.161 & 0.376 & 0.284 & 0.601 & 0.460 & & & & \\
\hline \multicolumn{13}{|l|}{ Completion } \\
\hline Voc/Cert & 0.372 & 0.273 & 0.484 & 0.408 & 0.557 & 0.499 & 0.595 & 0.538 & & & & \\
\hline $\mathrm{AA}$ & 0.167 & 0.140 & 0.281 & 0.226 & 0.386 & 0.313 & 0.472 & 0.407 & & & & \\
\hline BA & 0.457 & 0.387 & 0.528 & 0.440 & 0.595 & 0.509 & 0.669 & 0.553 & & & & \\
\hline
\end{tabular}


Table 3 Education and labor market outcomes: by hs achievement quartile (Continued)

\begin{tabular}{|c|c|c|c|c|c|c|c|c|}
\hline \multicolumn{9}{|l|}{ Intermediate HS outcomes } \\
\hline \multirow[t]{2}{*}{ 10th grade FCAT math score } & -1.189 & -1.310 & -0.184 & -0.208 & 0.376 & 0.348 & 1.148 & 1.035 \\
\hline & $(0.748)$ & $(0.813)$ & $(0.17)$ & $(0.171)$ & $(0.166)$ & $(0.163)$ & $(0.413)$ & $(0.333)$ \\
\hline \multirow[t]{2}{*}{ HS GPA } & 2.032 & 1.941 & 2.395 & 2.283 & 2.730 & 2.579 & 3.201 & 2.973 \\
\hline & $(0.676)$ & $(0.662)$ & $(0.64)$ & $(0.649)$ & $(0.624)$ & $(0.655)$ & $(0.569)$ & $(0.637)$ \\
\hline \multicolumn{9}{|l|}{$\begin{array}{l}\text { Intermediate postsecondary } \\
\text { outcomes }\end{array}$} \\
\hline \multirow[t]{2}{*}{ Postsecondary GPA } & 1.826 & 1.657 & 2.174 & 2.022 & 2.461 & 2.317 & 2.865 & 2.682 \\
\hline & $(1.070)$ & $(1.084)$ & $(0.981)$ & $(1.013)$ & $(0.913)$ & $(0.973)$ & $(0.793)$ & $(0.892)$ \\
\hline \multirow[t]{2}{*}{ Total postsecondary credits } & 46.025 & 41.735 & 64.693 & 58.914 & 82.596 & 75.059 & 103.629 & 91.618 \\
\hline & $(49.98)$ & $(48.566)$ & $(56.496)$ & $(56.072)$ & $(59.317)$ & $(60.512)$ & $(60.924)$ & $(62.933)$ \\
\hline \multirow[t]{2}{*}{ Postsecondary math credits } & 5.686 & 5.225 & 8.102 & 7.714 & 8.885 & 8.961 & 9.461 & 9.694 \\
\hline & $(7.693)$ & $(7.763)$ & $(7.972)$ & $(8.244)$ & $(7.529)$ & $(8.367)$ & $(8.551)$ & $(8.631)$ \\
\hline \multirow[t]{2}{*}{ Postsecondary science credits } & 5.847 & 5.214 & 9.162 & 8.472 & 12.863 & 12.042 & 17.580 & 16.601 \\
\hline & $(11.001)$ & $(10.675)$ & $(13.896)$ & $(14.353)$ & $(17.937)$ & $(18.004)$ & $(22.205)$ & $(22.442)$ \\
\hline \multicolumn{9}{|l|}{ Labor market inputs } \\
\hline \multirow[t]{2}{*}{ Work experience } & 19.562 & 18.636 & 19.676 & 18.873 & 19.778 & 18.859 & 18.910 & 18.505 \\
\hline & $(11.556)$ & $(11.36)$ & $(11.118)$ & $(10.987)$ & $(10.767)$ & $(10.743)$ & (9.978) & $(10.317)$ \\
\hline \multirow[t]{2}{*}{ Tenure } & 6.442 & 5.986 & 6.915 & 6.251 & 7.021 & 6.269 & 6.886 & 6.566 \\
\hline & (7.188) & $(6.715)$ & (7.387) & $(6.775)$ & (7.177) & (6.604) & (6.687) & (6.603) \\
\hline
\end{tabular}

Notes: Quartile 1 is the lowest achievement level, and quartile 4 is the highest achievement level. Educational attainment and enrollment are conditional upon HS completion, and completion is conditional upon enrollment in that degree. 
workers and students, then separately by race and FRL status respectively. In these tables, differences in outcomes observed between FCAT quartiles likely reflect the effects of selection by ability/achievement, while those differences within FCAT quartiles are likely attributable to other factors.

The results in Table 3A show large differences in both academic and labor market outcomes across FCAT quartiles. These differences are clearest and monotonic across achievement quartiles for earnings and tenure; BA attainment, enrollment and completion; and various measures of postsecondary achievement. In particular, BA enrollment and completion rates are heavily affected by selection on high school achievement. Enrollment in and completion of certificate and AA programs are less clearly affected by such selection.

Additionally, regardless of quartile, we find low completion rates in AA and BA programs. Specifically, conditional on enrollment, we find that $46 \%$ and $66 \%$ in the top quartile complete their AA and BA programs respectively; the comparable numbers in the second quartile are $26 \%$ and $50 \%$ respectively. These findings suggest that a range of other factors influence higher education outcomes as substantial numbers of even the highest achieving students fail to earn degrees.

These findings are generally confirmed in Tables $3 \mathrm{~B}$ and $\mathrm{C}$, which show the results broken out by race and FRL status. While differences in academic and labor market outcomes are partly accounted for by differences in high school achievement across these groups, important differences remain within particular levels of achievement. For instance, we find that black BA enrollments and postsecondary credits are often higher than those of whites within achievement quartiles, but their completion rates at all levels lag behind, as do their labor market earnings and attainment of work experience. In addition, FRL students lag behind non-FRL students in BA enrollments (and in AA enrollments below the top quartile), all intermediate postsecondary outcomes, and in earnings.

Within achievement quartiles, differences in educational attainment are large and quantitatively important, and we need to understand their determinants much better than we presently do.

\subsection{Fields of study}

It is well known that, for any level of academic attainment, labor market rewards differ significantly across fields of study (Altonji et al. 2012). In particular, the fields of science, technology, engineering and math (or STEM) are relatively highly rewarded.

In Table 4, we present the distributions of students across fields of study. We present these distributions separately for those in Vocational/Certificate, AA, and BA programs; and, within those, separately for completers and all attempters. If we compare ratios of concentration levels of completers to attempters by field, we can infer differential completion rates across these fields at each level of schooling. We present results for all students and workers, although results are similar by FRL status and race.

Among our results, we find that vocational certificate students tend to concentrate heavily in Health Technology and Security, and to a lesser extent Construction and Other Health, and their completion rates are high in all these categories. Students at the AA level are concentrated in Legal studies and especially Humanities, but completion rates are relatively low in the latter. At the BA level, concentrations are high in Business/ 
Table 4 Distribution of field of study by degree type and completion

\begin{tabular}{|c|c|c|c|c|c|c|}
\hline \multirow[b]{2}{*}{ Fields of study } & \multicolumn{2}{|c|}{ Voc/Cert } & \multicolumn{2}{|l|}{ AA } & \multicolumn{2}{|l|}{$B A$} \\
\hline & Comp. & $\overline{\text { Attempt }}$ & Comp. & $\overline{\text { Attempt }}$ & Comp. & Attempt \\
\hline Manufacturing & 0.007 & 0.009 & 0.001 & 0.001 & 0.000 & 0.000 \\
\hline Construction & 0.085 & 0.073 & 0.001 & 0.005 & 0.000 & 0.000 \\
\hline Health tech & 0.258 & 0.130 & 0.024 & 0.023 & 0.000 & 0.001 \\
\hline Other health & 0.100 & 0.066 & 0.055 & 0.072 & 0.078 & 0.068 \\
\hline Transportation & 0.004 & 0.004 & 0.002 & 0.001 & 0.000 & 0.000 \\
\hline Business/Management & 0.055 & 0.044 & 0.008 & 0.025 & 0.216 & 0.192 \\
\hline Education & 0.013 & 0.025 & 0.003 & 0.007 & 0.085 & 0.080 \\
\hline Engineering & 0.045 & 0.040 & 0.018 & 0.028 & 0.079 & 0.084 \\
\hline Communications & 0.005 & 0.003 & 0.003 & 0.004 & 0.060 & 0.050 \\
\hline Legal & 0.001 & 0.003 & 0.392 & 0.122 & 0.006 & 0.005 \\
\hline Security & 0.332 & 0.198 & 0.007 & 0.016 & 0.042 & 0.038 \\
\hline Bio, Math/Stats, Physical science & 0.005 & 0.004 & 0.002 & 0.003 & 0.069 & 0.078 \\
\hline Social science & 0.000 & 0.001 & 0.001 & 0.003 & 0.203 & 0.180 \\
\hline Humanities & 0.009 & 0.026 & 0.480 & 0.554 & 0.122 & 0.124 \\
\hline Other & 0.078 & 0.053 & 0.003 & 0.006 & 0.034 & 0.030 \\
\hline Missing & 0.002 & 0.322 & 0.001 & 0.131 & 0.006 & 0.072 \\
\hline $\mathrm{N}$ & 12,060 & 28,775 & 43,236 & 144,231 & 45,564 & 77,028 \\
\hline
\end{tabular}

Notes: "Comp." denotes completers and "Attempt" denotes all attempters of that degree, regardless of completion status. Each number represents the share of students attempting a given degree who completed or attempted (depending on column header) a given field. For example, of students who completed an AA degree, 48 percent of them were awarded degrees in Humanities; and of students who attempted an AA degree, 55.4 percent attempted a Humanities degree. Students who did not complete a degree are counted as attempting their final declared major prior to leaving postsecondary studies.

Management and the Social Sciences, and to a lesser extent in Other Health, Education, Engineering, Humanities, and Math/Physical Sciences; and completion rates are relatively low in Engineering, Math/Physical Sciences and the Humanities.

The low concentration in STEM fields by students in two-year institutions - who tend to come from disadvantaged backgrounds and/or with lower academic performance - may reflect rational decision-making, as STEM programs are associated with higher study times, harsher grading standards, and higher dropout rates for students with lower academic preparation (Arcidiacono et al. 2012; Arcidiacono et al. 2013). On the other hand, their relatively high concentration in other low-completion areas, like Humanities at the AA level (which is almost always "liberal arts" or "general studies" and designed to lead to transfer to four-year institution), is potentially more troubling given the low returns to these degrees, as shown in the next section.

\subsection{Regression results}

Tables 5, 6 and 7 show the results of estimated versions of Equation 1. The dependent variable is the natural log of quarterly earnings, and the sample is limited to those 18 years of age and older who have exited, or never entered, postsecondary education. The wage data are available for 5 years following a student's last educational enrollment ${ }^{17}$.

Five specifications of Equation 1 appear in Table 5. The first includes dummy variables for highest educational attainment, quarters of work experience and tenure with the current employer (in linear and quadratic form) ${ }^{18}$, a cubic polynomial of high school math 
Table 5 Regression results for log quarterly earnings: all workers

\begin{tabular}{|c|c|c|c|c|c|}
\hline & $(1)$ & $(2)$ & $(3)$ & (4) & $(5)$ \\
\hline \multicolumn{6}{|l|}{ Highest credential } \\
\hline \multirow[t]{2}{*}{ No HS } & $-0.15^{* * *}$ & $-0.13^{* * *}$ & $-0.11^{* * *}$ & $-0.11^{* * *}$ & $-0.11^{* * *}$ \\
\hline & $(0.00)$ & $(0.00)$ & $(0.00)$ & $(0.00)$ & $(0.00)$ \\
\hline \multirow[t]{2}{*}{ Voc_Cert } & $0.31^{* * *}$ & $0.26^{* * *}$ & $0.29^{* * *}$ & $0.18^{* * *}$ & $0.18^{* * *}$ \\
\hline & $(0.01)$ & $(0.01)$ & $(0.01)$ & $(0.01)$ & $(0.01)$ \\
\hline \multirow[t]{2}{*}{ AA } & $0.34^{* * *}$ & $0.36^{* * *}$ & $0.40^{* * *}$ & $0.10^{* * *}$ & $0.02^{* *}$ \\
\hline & $(0.01)$ & $(0.01)$ & $(0.01)$ & $(0.01)$ & $(0.01)$ \\
\hline \multirow[t]{2}{*}{ AS } & & & & & $0.29^{* * *}$ \\
\hline & & & & & $(0.02)$ \\
\hline \multirow[t]{2}{*}{ BA } & $0.61^{* * *}$ & $0.69^{* * *}$ & $0.78^{* * *}$ & $0.39^{* * *}$ & $0.39^{* * *}$ \\
\hline & $(0.01)$ & $(0.01)$ & $(0.01)$ & $(0.01)$ & $(0.01)$ \\
\hline \multirow[t]{2}{*}{ MA_PhD } & $0.89^{* * *}$ & $0.99^{* * *}$ & $1.08^{* * *}$ & $0.66^{* * *}$ & $0.66^{* * *}$ \\
\hline & $(0.01)$ & $(0.01)$ & $(0.01)$ & $(0.02)$ & $(0.02)$ \\
\hline \multirow[t]{2}{*}{ Work experience } & $0.02^{* * *}$ & $0.02^{* * *}$ & $0.02^{* * *}$ & $0.02^{* * *}$ & $0.02^{* * *}$ \\
\hline & $(0.00)$ & $(0.00)$ & $(0.00)$ & $(0.00)$ & $(0.00)$ \\
\hline \multirow[t]{2}{*}{ Work experience ${ }^{2}$} & $-0.00^{* * *}$ & $-0.00^{* * *}$ & $-0.00 * * *$ & $-0.00^{* * *}$ & $-0.00^{* * *}$ \\
\hline & $(0.00)$ & $(0.00)$ & $(0.00)$ & $(0.00)$ & $(0.00)$ \\
\hline \multirow[t]{2}{*}{ Tenure } & $0.08^{* * *}$ & $0.08^{* * *}$ & $0.08^{* * *}$ & $0.08^{* * *}$ & $0.08^{* * *}$ \\
\hline & $(0.00)$ & $(0.00)$ & $(0.00)$ & $(0.00)$ & $(0.00)$ \\
\hline \multirow[t]{2}{*}{ Tenure $^{2}$} & $-0.00^{* * *}$ & $-0.00^{* * *}$ & $-0.00^{* * *}$ & $-0.00^{* * *}$ & $-0.00^{* * *}$ \\
\hline & $(0.00)$ & $(0.00)$ & $(0.00)$ & $(0.00)$ & $(0.00)$ \\
\hline \multirow[t]{2}{*}{ FCAT } & $0.07^{* * *}$ & $0.06^{* * *}$ & $0.06^{* * *}$ & $0.04^{* * *}$ & $0.04^{* * *}$ \\
\hline & $(0.00)$ & $(0.00)$ & $(0.00)$ & $(0.00)$ & $(0.00)$ \\
\hline \multirow[t]{2}{*}{ Female } & $-0.15^{* * *}$ & $-0.15^{* * *}$ & $-0.15^{* * *}$ & $-0.16^{* * *}$ & $-0.16^{* * *}$ \\
\hline & $(0.00)$ & $(0.00)$ & $(0.00)$ & $(0.00)$ & $(0.00)$ \\
\hline \multirow[t]{2}{*}{ FRL } & $-0.07^{* * *}$ & $-0.07^{* * *}$ & $-0.06^{* * *}$ & $-0.06^{* * *}$ & $-0.06^{* * *}$ \\
\hline & $(0.00)$ & $(0.00)$ & $(0.00)$ & $(0.00)$ & $(0.00)$ \\
\hline \multirow[t]{2}{*}{ Black } & $-0.11^{* * *}$ & $-0.12^{* * *}$ & $-0.12^{* * *}$ & $-0.11^{* * *}$ & $-0.11^{* * *}$ \\
\hline & $(0.00)$ & $(0.00)$ & $(0.00)$ & $(0.00)$ & $(0.00)$ \\
\hline \multirow[t]{2}{*}{ Hispanic } & $0.16^{* * *}$ & $0.15^{* * *}$ & $0.14^{* * *}$ & $0.15^{* * *}$ & $0.15^{* * *}$ \\
\hline & $(0.00)$ & $(0.00)$ & $(0.00)$ & $(0.00)$ & $(0.00)$ \\
\hline \multirow[t]{2}{*}{ Asian } & $0.08^{* * *}$ & $0.07^{* * *}$ & $0.07^{* * *}$ & $0.06^{* * *}$ & $0.06^{* * *}$ \\
\hline & $(0.01)$ & $(0.01)$ & $(0.01)$ & $(0.01)$ & $(0.01)$ \\
\hline \multirow[t]{2}{*}{ Other } & $-0.08^{* * *}$ & $-0.08^{* * *}$ & $-0.08^{* * *}$ & $-0.08^{* * *}$ & $-0.08^{* * *}$ \\
\hline & $(0.02)$ & $(0.01)$ & $(0.01)$ & $(0.01)$ & $(0.01)$ \\
\hline \multicolumn{6}{|l|}{ CC Credits } \\
\hline \multirow[t]{2}{*}{0 credits } & & & $0.20^{* * *}$ & & \\
\hline & & & $(0.01)$ & & \\
\hline \multirow[t]{2}{*}{$1-15$ credits } & & & $0.28^{* * *}$ & & \\
\hline & & & $(0.01)$ & & \\
\hline \multirow[t]{2}{*}{$16-30$ credits } & & & $0.28^{* * *}$ & & \\
\hline & & & $(0.01)$ & & \\
\hline \multirow[t]{2}{*}{$31-45$ credits } & & & $0.30^{* * *}$ & & \\
\hline & & & $(0.01)$ & & \\
\hline
\end{tabular}


Table 5 Regression results for log quarterly earnings: all workers (Continued)

\begin{tabular}{|c|c|c|c|c|c|}
\hline \multirow[t]{2}{*}{$46-60$ credits } & & & $0.32^{* * *}$ & & \\
\hline & & & $(0.01)$ & & \\
\hline \multirow[t]{2}{*}{$60-80$ credits } & & & $0.32^{* * *}$ & & \\
\hline & & & $(0.01)$ & & \\
\hline \multicolumn{6}{|l|}{ University credits } \\
\hline \multirow[t]{2}{*}{0 credits } & & & 0.02 & & \\
\hline & & & $(0.03)$ & & \\
\hline \multirow[t]{2}{*}{$1-30$ credits } & & & $0.26^{* * *}$ & & \\
\hline & & & $(0.01)$ & & \\
\hline \multirow[t]{2}{*}{$31-60$ credits } & & & $0.34^{* * *}$ & & \\
\hline & & & $(0.01)$ & & \\
\hline \multirow[t]{2}{*}{$61-90$ credits } & & & $0.40^{* * *}$ & & \\
\hline & & & $(0.02)$ & & \\
\hline \multirow[t]{2}{*}{$91-120$ credits } & & & $0.48^{* * *}$ & & \\
\hline & & & $(0.03)$ & & \\
\hline \multirow[t]{2}{*}{$121-150$ credits } & & & $0.55^{* * *}$ & & \\
\hline & & & $(0.03)$ & & \\
\hline \multicolumn{6}{|c|}{ Post-secondary credits earned (100s) } \\
\hline \multirow[t]{2}{*}{ CC credits } & & $0.57^{* * *}$ & & $0.21^{* * *}$ & $0.20^{* * *}$ \\
\hline & & $(0.01)$ & & $(0.01)$ & $(0.01)$ \\
\hline \multirow[t]{2}{*}{ University credits } & & $0.47^{* * *}$ & & $0.23^{* * *}$ & $0.24^{* * *}$ \\
\hline & & $(0.01)$ & & $(0.01)$ & $(0.01)$ \\
\hline \multirow[t]{2}{*}{ Post-secondary GPA } & & & & $0.10^{* * *}$ & $0.10^{* * *}$ \\
\hline & & & & $(0.00)$ & $(0.00)$ \\
\hline \multicolumn{6}{|c|}{ Post-secondary credits (10s) } \\
\hline \multirow[t]{2}{*}{ Math } & & & & $0.04^{* * *}$ & $0.05^{* * *}$ \\
\hline & & & & $(0.00)$ & $(0.00)$ \\
\hline \multirow[t]{2}{*}{ Science } & & & & $0.02^{* * *}$ & $0.02^{* * *}$ \\
\hline & & & & $(0.00)$ & $(0.00)$ \\
\hline Observations & $3,739,354$ & $3,739,354$ & $3,739,354$ & $3,739,354$ & $3,739,354$ \\
\hline R-squared & 0.36 & 0.36 & 0.36 & 0.36 & 0.36 \\
\hline
\end{tabular}
scale. Quadratic and cubic FCAT terms are estimated but not reported.

FCAT score (to control for academic selection effects) ${ }^{19}$, and demographic dummy variables (race, gender, and FRL eligibility). The second equation adds the number of credits earned at either a two- or four-year school for students who enrolled but did not complete a degree. The third equation allows for non-linear returns to college credits by allowing the return to vary by the number of credits earned ${ }^{20}$. In the fourth equation, we add additional controls for postsecondary academic achievement: GPA and numbers of postsecondary courses taken in math and science. In the fifth equation, the dummy variable for AA degrees is split into those obtaining an Associate of Applied Science (AAS) or Associate of Science (AS) degree versus all others.

Several important findings appear in Table 5. As expected, we find strong labor market returns on average to all postsecondary credentials. Specifically, those obtaining 


\begin{tabular}{|c|c|c|}
\hline & AA \& below & BA \& above \\
\hline \multicolumn{3}{|l|}{ Highest credential } \\
\hline \multirow[t]{2}{*}{ No HS } & $-0.12^{* * *}$ & \\
\hline & $(0.01)$ & \\
\hline \multirow[t]{2}{*}{ Voc_Cert } & -0.05 & $0.40^{* * *}$ \\
\hline & $(0.12)$ & $(0.09)$ \\
\hline \multirow[t]{2}{*}{ AA } & 0.19 & $0.31^{* * *}$ \\
\hline & $(0.12)$ & $(0.07)$ \\
\hline \multirow[t]{2}{*}{ BA } & & $0.48^{* * *}$ \\
\hline & & $(0.07)$ \\
\hline \multirow[t]{2}{*}{ MA_PhD } & & $0.73^{* * *}$ \\
\hline & & $(0.07)$ \\
\hline \multicolumn{3}{|l|}{ Completed field of study } \\
\hline Manufacturing & $0.26^{* * *}$ & $-1.87^{* * *}$ \\
\hline Construction & $0.25^{* * *}$ & $0.29^{*}$ \\
\hline Health Tech & $0.23^{* * *}$ & $0.16^{* *}$ \\
\hline Other Health & $0.48^{* * *}$ & $0.39^{* * *}$ \\
\hline Transportation & $0.30^{* *}$ & $0.81^{* * *}$ \\
\hline Business/Management & $0.08^{*}$ & $0.29^{* * *}$ \\
\hline Education & $-0.38^{* * *}$ & $0.03^{*}$ \\
\hline Engineering & 0.06 & $0.47^{* * *}$ \\
\hline Communications & $-0.21^{* *}$ & $0.15^{* * *}$ \\
\hline Legal & -0.01 & $0.10^{* * *}$ \\
\hline Security & $0.32^{* * *}$ & $0.07^{* * *}$ \\
\hline Bio, Math/Stats, Phys Sci & $0.15^{* *}$ & $0.20^{* * *}$ \\
\hline Social Science & $-0.80^{* * *}$ & $0.07^{* * *}$ \\
\hline Other & $-0.08^{*}$ & $0.13^{* * *}$ \\
\hline No CIP & 0.02 & $0.31^{* * *}$ \\
\hline \multirow[t]{2}{*}{ Work experience } & $0.02^{* * *}$ & $0.02^{* * *}$ \\
\hline & $(0.00)$ & $(0.00)$ \\
\hline \multirow[t]{2}{*}{ Work experience ${ }^{2}$} & $-0.00^{* * *}$ & $-0.00^{* * *}$ \\
\hline & $(0.00)$ & $(0.00)$ \\
\hline \multirow[t]{2}{*}{ Tenure } & $0.08^{* * *}$ & $0.05^{* * *}$ \\
\hline & $(0.00)$ & $(0.00)$ \\
\hline \multirow[t]{2}{*}{ Tenure $^{2}$} & $-0.00^{* * *}$ & $-0.00 * * *$ \\
\hline & $(0.00)$ & $(0.00)$ \\
\hline \multirow[t]{2}{*}{ FCAT } & $0.06^{* * *}$ & $0.07^{* * *}$ \\
\hline & $(0.01)$ & $(0.01)$ \\
\hline \multirow[t]{2}{*}{ Female } & $-0.13^{* * *}$ & $-0.08^{* * *}$ \\
\hline & $(0.01)$ & $(0.01)$ \\
\hline \multirow[t]{2}{*}{ FRL } & $-0.05^{* * *}$ & $-0.02^{*}$ \\
\hline & $(0.01)$ & $(0.01)$ \\
\hline \multirow[t]{2}{*}{ Black } & $-0.08^{* * *}$ & $-0.07^{* * *}$ \\
\hline & $(0.01)$ & $(0.01)$ \\
\hline
\end{tabular}


Table 6 Regression results for log quarterly earnings by fields of study and level of education (Continued)

\begin{tabular}{|c|c|c|}
\hline \multirow[t]{2}{*}{ Hispanic } & $0.15^{* * *}$ & $0.08^{* * *}$ \\
\hline & $(0.01)$ & $(0.01)$ \\
\hline \multirow[t]{2}{*}{ Asian } & 0.03 & -0.01 \\
\hline & $(0.02)$ & $(0.02)$ \\
\hline \multirow[t]{2}{*}{ Other } & $-0.08^{* *}$ & -0.01 \\
\hline & $(0.03)$ & $(0.05)$ \\
\hline Observations & 635,272 & 264,516 \\
\hline R-squared & 0.24 & 0.19 \\
\hline
\end{tabular}

a vocational certificate earn approximately 30 percent more per quarter than high school graduates; those earning AA degrees earn 35-40 percent more than high school graduates - though returns to AAS/AS degrees are much higher than those for other AAs (in Column 5); and the BA degree earns $60-80$ percent more per quarter, which is about double what AA degrees earn, and those with graduate degrees earn considerably more $^{21}$. Our estimates of returns to these credentials are at the high end of the range found in the other recent studies using state-specific administrative data and are a bit higher than (but still qualitatively consistent with) those estimated recently using national survey data.

The results in the second and third equations indicate that there are also returns, on average, to attending a program and earning credits, even if the program is not completed. In addition, there are larger returns (relative to those with no postsecondary enrollment) to accumulating more credits $^{22}$. But these returns are smaller than those for completed degrees, indicating a "sheepskin effect," especially for bachelor's degrees. The magnitudes are broadly consistent with others who have found evidence of these effects in the literature (e.g., Kane and Rouse, Jepsen et al., Kreisman et al., op. cit.) ${ }^{23}$. Furthermore, since most dropouts accumulate many fewer credits than program completers, the dropouts are relatively hurt both by their fewer credits and by the absence of the formal credential. Thus, the low levels of completion observed in AA programs, and the gaps between race, income, and achievement groups at the BA level all reduce the future earnings of minority and low-income students.

There is also a very strong return to postsecondary GPA and also a return to taking math and science courses. When these variables are added to the equation, the estimated returns to completed degrees drop substantially, which indicates that at least some part of the observed returns to degree programs are really for overall achievement and technical skills ${ }^{24}$. On the other hand, it could also be the case that high postsecondary GPA captures unmeasured ability in a way our other variables do not, thus explaining additional selection into degree program. The relatively high returns to mostly technical certificate programs, and to AAS/AS degrees rather than AAs, confirm that the market returns to technical skills, including at the sub-BA level, are relatively large ${ }^{25}$.

A few other findings in Table 5 are noteworthy. First, the returns to general work experience are modest, but in contrast, the returns to tenure are quite substantial, with 
Table 7 Regression results for log quarterly earnings by demographic and fcat groups

\begin{tabular}{|c|c|c|c|c|c|c|}
\hline & \multicolumn{2}{|l|}{ Gender } & \multicolumn{2}{|l|}{$\underline{F R L}$} & \multicolumn{2}{|l|}{ FCAT } \\
\hline & Male & Female & No & Yes & Top half & Bottom half \\
\hline \multicolumn{7}{|l|}{ Highest credential } \\
\hline \multirow[t]{2}{*}{ No HS } & $-0.12^{* * *}$ & $-0.19^{* * *}$ & $-0.14^{* * *}$ & $-0.16^{* * *}$ & $-0.11^{* * *}$ & $-0.15^{* * *}$ \\
\hline & $(0.00)$ & $(0.00)$ & $(0.00)$ & $(0.00)$ & $(0.01)$ & $(0.00)$ \\
\hline \multirow[t]{2}{*}{ Voc_Cert } & $0.35^{* * *}$ & $0.23^{* * *}$ & $0.29^{* * *}$ & $0.35^{* * *}$ & $0.24^{* * *}$ & $0.34^{* * *}$ \\
\hline & $(0.01)$ & $(0.02)$ & $(0.01)$ & $(0.02)$ & $(0.02)$ & $(0.01)$ \\
\hline \multirow[t]{2}{*}{$\mathrm{AA}$} & $0.29^{* * *}$ & $0.35^{* * *}$ & $0.31^{* * *}$ & $0.40^{* * *}$ & $0.25^{* * *}$ & $0.42^{* * *}$ \\
\hline & $(0.01)$ & $(0.01)$ & $(0.01)$ & $(0.02)$ & $(0.01)$ & $(0.01)$ \\
\hline \multirow[t]{2}{*}{$\mathrm{BA}$} & $0.64^{* * *}$ & $0.57^{* * *}$ & $0.59^{* * *}$ & $0.68^{* * *}$ & $0.51^{* * *}$ & $0.69^{* * *}$ \\
\hline & $(0.01)$ & $(0.01)$ & $(0.01)$ & $(0.01)$ & $(0.01)$ & $(0.01)$ \\
\hline \multirow[t]{2}{*}{ MA_PhD } & $1.03^{* * *}$ & $0.81^{* * *}$ & $0.86^{* * *}$ & $1.01 * * *$ & $0.80^{* * *}$ & $0.96^{* * *}$ \\
\hline & $(0.02)$ & $(0.02)$ & $(0.01)$ & $(0.04)$ & $(0.02)$ & $(0.03)$ \\
\hline \multirow[t]{2}{*}{ Work experience } & $0.02^{* * *}$ & $0.02^{* * *}$ & $0.02^{* * *}$ & $0.02^{* * *}$ & $0.02^{* * *}$ & $0.02^{* * *}$ \\
\hline & $(0.00)$ & $(0.00)$ & $(0.00)$ & $(0.00)$ & $(0.00)$ & $(0.00)$ \\
\hline \multirow[t]{2}{*}{ Work experience ${ }^{2}$} & $-0.00^{* * *}$ & $-0.00^{* * *}$ & $-0.00^{* * *}$ & $-0.00^{* * *}$ & $-0.00^{* * *}$ & $-0.00^{* * *}$ \\
\hline & $(0.00)$ & $(0.00)$ & $(0.00)$ & $(0.00)$ & $(0.00)$ & $(0.00)$ \\
\hline \multirow[t]{2}{*}{ Tenure } & $0.08^{* * *}$ & $0.07^{* * *}$ & $0.07^{* * *}$ & $0.08^{* * *}$ & $0.06^{* * *}$ & $0.08^{* * *}$ \\
\hline & $(0.00)$ & $(0.00)$ & $(0.00)$ & $(0.00)$ & $(0.00)$ & $(0.00)$ \\
\hline \multirow[t]{2}{*}{ Tenure $^{2}$} & $-0.00^{* * *}$ & $-0.00^{* * *}$ & $-0.00^{* * *}$ & $-0.00^{* * *}$ & $-0.00^{* * *}$ & $-0.00^{* * *}$ \\
\hline & $(0.00)$ & $(0.00)$ & $(0.00)$ & $(0.00)$ & $(0.00)$ & $(0.00)$ \\
\hline \multirow[t]{2}{*}{ FCAT } & $0.05^{* * *}$ & $0.09^{* * *}$ & $0.07^{* * *}$ & $0.07^{* * *}$ & $0.13^{* * *}$ & $0.07^{* * *}$ \\
\hline & $(0.00)$ & $(0.00)$ & $(0.00)$ & $(0.00)$ & $(0.02)$ & $(0.02)$ \\
\hline \multirow[t]{2}{*}{ FRL } & $-0.06^{* * *}$ & $-0.09^{* * *}$ & & & $-0.06^{* * *}$ & $-0.07^{* * *}$ \\
\hline & $(0.00)$ & $(0.00)$ & & & $(0.01)$ & $(0.00)$ \\
\hline \multirow[t]{2}{*}{ Female } & & & $-0.15^{* * *}$ & $-0.15^{* * *}$ & $-0.13^{* * *}$ & $-0.15^{* * *}$ \\
\hline & & & $(0.00)$ & $(0.00)$ & $(0.00)$ & $(0.00)$ \\
\hline \multirow[t]{2}{*}{ Black } & $-0.17^{* * *}$ & $-0.03^{* * *}$ & $-0.13^{* * *}$ & $-0.08^{* * *}$ & $-0.07^{* * *}$ & $-0.12^{* * *}$ \\
\hline & $(0.00)$ & $(0.00)$ & $(0.00)$ & $(0.00)$ & $(0.01)$ & $(0.00)$ \\
\hline \multirow[t]{2}{*}{ Hispanic } & $0.11^{* * *}$ & $0.21^{* * *}$ & $0.11^{* * *}$ & $0.20^{* * *}$ & $0.13^{* * *}$ & $0.16^{* * *}$ \\
\hline & $(0.00)$ & $(0.00)$ & $(0.00)$ & $(0.00)$ & $(0.01)$ & $(0.00)$ \\
\hline \multirow[t]{2}{*}{ Asian } & $0.03^{* *}$ & $0.13^{* * *}$ & $0.05^{* * *}$ & $0.13^{* * *}$ & $0.07^{* * *}$ & $0.07^{* * *}$ \\
\hline & $(0.01)$ & $(0.02)$ & $(0.01)$ & $(0.02)$ & $(0.02)$ & $(0.01)$ \\
\hline \multirow[t]{2}{*}{ Other } & $-0.14^{* * *}$ & -0.00 & $-0.10^{* * *}$ & $-0.05^{* *}$ & -0.03 & $-0.10^{* * *}$ \\
\hline & $(0.02)$ & $(0.02)$ & $(0.02)$ & $(0.02)$ & $(0.03)$ & $(0.02)$ \\
\hline Observations & $2,096,183$ & $1,656,973$ & $2,196,457$ & $1,556,699$ & $1,052,977$ & $2,700,179$ \\
\hline R-squared & 0.35 & 0.37 & 0.36 & 0.33 & 0.35 & 0.33 \\
\hline
\end{tabular}

Notes: ${ }^{* * *} \mathrm{p}<0.01,{ }^{* *} \mathrm{p}<0.05,{ }^{*} \mathrm{p}<0.1$. The dependent variable is $\ln$ (quarterly earnings). Robust standard errors are in parentheses. FCAT scores are standardized to mean 1, standard deviation 0. Quadratic and cubic FCAT terms are estimated but not reported.

a first quarter return of about eight percent. Thus, accumulating job tenure for a year or more is quite substantially rewarded. Though we made no effort here to measure returns to work experience specifically tied to one's postsecondary degree - which presumably are much greater than these - the results indicate that accumulating some kinds of work experience before, during or after one's postsecondary schooling can be 
valuable. In addition, the returns to FCAT indicate some significant academic selection effects, which we control for in our analysis ${ }^{26}$.

Finally, we note the coefficients on demographic variables in these equations. Interestingly, controlling for the full range of academic attainment and achievement generates even larger negative effects for females - of 15 percent per quarter - than we found in Table 1, since their achievement measures are generally higher than those of males, while their earnings are lower. Controls for education and achievement also generate large (15 percent) returns for Hispanics relative to whites. And, for blacks and low-income students, estimated negative effects are now much smaller (6 to 12 percent per quarter) than we observed in Table 1, indicating that much (though not all) of their lower earnings is associated with lower academic attainment and achievement ${ }^{27}$.

Despite our extensive controls for demographic information and achievement in high school, it is still possible that our estimates reflect selection on unobserved factors. For instance, for two students with the same demographic information and performance in high school who choose two different postsecondary paths and have different labor market outcomes, we cannot say for certain whether it was the paths that caused the disparate outcomes or some unobserved factor such as motivation or guidance from others. On the other hand, including controls for high school achievement generates only modest changes in our estimated coefficients on schooling, relative to equations which included no such controls. Thus, it seems unlikely that other unobservable characteristics have substantial effects on these estimates ${ }^{28}$.

\subsection{Specific fields, specific groups}

To further explore how these average effects on earnings vary with field of study, we present returns to different fields in Table 6. These estimates are from versions of Equation 1 containing dummy variables for fields of study, with Humanities as the omitted group. In separately estimated equations, we present results for those earning AAs or certificates and those earnings BAs.

The results show substantial variation in returns across fields of study. In particular, those earning sub-BA credentials have relatively strong returns to health, transportation, construction, manufacturing (mostly certificates), and security credentials; and for those earning BAs or higher, returns are strongest in transportation, engineering, business management and health, with smaller but still positive pay premia (relative to humanities) in the math/science, communications, legal work, and health technology ${ }^{29}$.

Importantly, the returns to the omitted category of AAs and humanities - the most commonly pursued degree in two-year colleges and also a field with relatively low completion rates - are also relatively low, compared to virtually all other fields. A strong case can be made therefore that the large concentrations of $A A$ students in these low completion and low return areas are sub-optimal, at least in terms of future earnings potential.

Our final research question is how these market returns vary across different demographic groups of students or those with varying levels of earlier achievement. To answer this question, we estimate the model (also based on the first equation in Table 5) separately for males and females, those eligible or not eligible for FRL, and those in the top or bottom half of FCAT scores ${ }^{30}$. These estimates appear in Table 7.

Overall, the patterns of market returns to postsecondary attainment and achievement are quite similar across demographic and achievement groups. A few modest differences 
can be found. For instance, men earn relatively larger rewards than women, both in certificate programs and BAs and above, while women do better in AA programs. In addition, academic credentials, including vocational certificates and AAs, are also relatively well-rewarded for FRL students and those students in the bottom half of high school achievers.

Thus, labor market rewards are broadly similar across these groups. But, in some more technical fields, achieving a vocational certificate or even an AA can be quite rewarding, especially among lower-achieving groups, and particularly if they can combine these credentials with good work experience or tenure afterwards.

\section{Conclusion}

Our paper examines a range of issues focusing on postsecondary education and labor market outcomes, with a particular focus on minorities and/or disadvantaged workers. We use administrative data from the state of Florida, where secondary and postsecondary education records for two cohorts of students have been linked to UI earnings data (for five years after schooling has been completed). The administrative data give us enormous samples with which to study particular groups of students, with very detailed information on educational experiences and outcomes for every public school student in a particular year. In addition, the secondary education records to which we have access enable us to test and control for selection based on early academic experience and achievement to a much greater extent than has been the case with other studies using administrative data. However, the data have their limitations, for instance, we cannot follow those who leave the state of Florida to attend college or enter the labor force. Yet, the richness of the data on educational experiences and outcomes, coupled with the recentness of the cohort and our large sample size, enable us to learn a great deal about postsecondary outcomes and their determinants.

Our main findings can be summarized as follows:

1) Variation in secondary school achievement can account for a large part of the gap in postsecondary attainment (through the differences we observe in enrollment rates and especially in completion) and labor market outcomes between racial and family income groups, but quite large differences also exist within achievement groups;

2) Earnings of minorities and disadvantaged students are reduced not only by their lower educational attainment and completion but also by weaker postsecondary academic performance and by their chosen fields of study.

More specifically, significant labor market premia are available in a variety of more technical certificate and AA programs, even for those with weak earlier academic performance. However, many disadvantaged (and other) students end up in general humanities (or liberal studies) programs at the AA (and even the BA) level, with low completion rates and low compensation afterwards.

Of course, students in liberal arts curricula, and especially those planning on post-BA education, are not necessarily harmed by majoring in humanities. And in none of this work can we control for students' preferences across fields; therefore, we cannot infer whether these outcomes reflect sub-optimal choices on the part of students. 
On the other hand, it is likely that many students in AA programs, especially disadvantaged students or those with weaker academic performance, do not plan on obtaining post-BA education and are hoping that their college experiences lead directly to higher earnings. For these students, the very high concentrations we observe in humanities programs at the AA level do not appear consistent with those goals.

One can imagine a variety of reallocations of students from these programs that would improve expected student outcomes, especially among the disadvantaged. These reallocations might include moving the higher achievers in this group to BA programs (especially at more selective institutions where completion rates are relatively high) or to more technical AA fields of study with higher completion rates and earnings; while lower achievers might do better in some of the well-compensated vocational certificate programs. Additionally, certificate programs and work experience are particularly wellcompensated among young men, especially African-American men who have difficulty gaining work experience and whose postsecondary attainments also lag substantially behind those of women.

How might such reallocations be accomplished? Poor choices by postsecondary students likely reflect at least two problems: poor information among students and poor incentives faced by their postsecondary institutions. Due to an unstructured environment and poor counseling, students in community college receive very little information about either academic or job market opportunities. Improving the guidance provided to these students would likely improve their outcomes (Scott-Clayton 2011; Jenkins and Cho 2012; Rosenbaum et al. 2006; Jacobson and Mokher 2009). But public institutions also need more incentive to respond to labor market factors and prepare students for well-paying or higher-demand fields, even if the costs to them of instruction and equipment in these fields are higher (Holzer 2014). Incentivizing these institutions to build more partnerships with industry associations, generating sectorial and career pathway programs, and helping students participate in them could be accomplished by greater use of postsecondary and earnings performance measures in determining state subsidies for public colleges and universities ${ }^{31}$.

In addition to these approaches, a few other broad policy guidelines are at least consistent with, and are perhaps suggested by, our findings. In particular, disadvantaged students would benefit from reforms in support programs and services, such as developmental (or remedial) education and financial aid ${ }^{32}$. Students might also benefit from work-based learning models and other pathways to postsecondary education, such as apprenticeships and other forms of high-quality career education, which do not substitute work experience for postsecondary learning but complement it and lead to improved labor market outcomes ${ }^{33}$. Experimentation with, and further evaluation of reforms in these support services and alternative pathways to skill creation should be a high priority.

\section{Endnotes}

${ }^{1}$ See Jacobson and Mokher (2009), Jepsen et al. (2014) and Kreisman et al. (2013).

${ }^{2}$ The unemployment insurance records do not include information on several small categories of employees including self-employed and federal workers; but they cover roughly $96 \%$ of all workers (Andersson et al. 2005).

${ }^{3}$ Institute of Education Sciences (2014). Digest of Education Statistics, Table 232. Retrieved May 21, 2014, from http://nces.ed.gov/programs/digest/d11/tables/dt11_232.asp 
${ }^{4}$ Institute of Education Sciences. Digest of Education Statistics, Table 233. Retrieved May 21, 2014, from http://nces.ed.gov/programs/digest/d12/tables/dt12_233.asp.

${ }^{5}$ Malamud and Wozniak (2008).

${ }^{6}$ For instance, in our oldest cohort, over $70 \%$ of those in the top achievement quartile appear in the labor market, whereas for the lowest quartile the comparable fraction is just $35 \%$. We examine the robustness of our results to including those with zero earnings for very limited numbers of quarters (see footnote 9), but we cannot address those with longer periods of no earnings (who might have left the state or left the labor market for a variety of reasons). The omission of these nonparticipants from our findings might bias downwards our estimates of returns to postsecondary credentials since the nonparticipants are likely high school graduates and dropouts who would have had low earnings had they worked. The same downward bias likely results from the omission of higher achievers and earners who moved out of state.

${ }^{7}$ In national data, the percentages of enrollees at community and public four-year college who are above the age of 24 are 40 and 21 percent, respectively (National Center for Education Statistics 2013, Table 303.50 of Digest 2013).

${ }^{8}$ Our measures of work experience and tenure do not count intermittent quarters with zero employment towards those measures.

${ }^{9}$ Researchers generally attribute quarters with zero earnings to labor supply decisions in which the individual did not choose to join the labor force or could not find employment; however, in our data, another possible explanation is that the individual moved out of state. To assess whether including short-term non-working spells are driving our results, we conduct a robustness check in which we impute earnings for individuals with 1-3 quarters of missing UI records in between observed employment spells. The results are robust to this imputation of zero earnings.

${ }^{10}$ While it is still possible that differences in outcomes within quartiles could reflect differences in achievement given the broadness of the quartiles, we use a variety of specifications for achievement measures in our earnings regressions below, including decile dummies, and find quite similar effects.

${ }^{11}$ In our descriptive tables, we pool whites and Asians, though in our regression estimates below we separate them (whites are the omitted group and Asians are indicated by a dummy variable). Though Asians earn more than whites, even controlling for education and achievement, their numbers are too small to generate major inconsistencies between the earlier descriptive results and our regression estimates.

${ }^{12}$ Regression results are qualitatively very similar when controlling for high school GPA and FCAT reading scores in addition to FCAT math scores. However, high school GPA is missing for one of our cohorts, so we choose not to include it (the correlation between FCAT math and high school GPA is just above 0.7). Since adding reading FCAT scores generates virtually identical results when already controlling for math FCAT scores, we focus only on math scores for simplicity.

${ }^{13} \mathrm{We}$ present quarterly, rather than annualized, earnings to be consistent with previous studies (e.g., Jepsen et al. 2014). To eliminate outliers, we limited our sample of quarterly earnings between $\$ 100$ and $\$ 100,000$.

${ }^{14}$ Swanson (2004) presents high school dropout rates using administrative data, though Mishel and Roy (2004) compare them to survey-based estimates, which are 
much higher. Using either method, Murnane (2013) shows a large decline in the estimated dropout rate over the past decade.

${ }^{15}$ When examining Hispanics separately by subgroup in the subsequent earnings regressions, relative to whites, all Hispanic groups earned more on average. The highest Hispanic earners are Cuban-born (about \$1,200 extra per quarter) and lowest earners are Puerto Rican-born (\$170 extra per quarter).

${ }^{16}$ While surveys tend to undercount low-income men, especially those who have been previously incarcerated or who are only marginally attached to households, the administrative data will undercount men or women whose employment is sporadic and informal, especially those paid in cash.

${ }^{17}$ By focusing on students' earnings after their education has been completed, we avoid confounding those with periods of lower earnings before or during the attainment of postsecondary credentials.

${ }^{18} \mathrm{Job}$ tenure and experience are likely endogenous as they are partially driven by postsecondary choices and outcomes. Results are qualitatively similar when not controlling for tenure and/or experience, although not controlling for either somewhat depresses the coefficient on BA completion since BA recipients in each cohort tend to have had less time in the labor market and thus lower earnings (conditional on degree earned) relative to others of the same age who have accrued more labor market experience.

${ }^{19}$ Variables with missing values for all of these measures are used in the regressions, with zeroes imputed for the missing values and dummy variables used to denote such cases. Results are robust to using a linear term in math FCAT scores or including a dummy variable for each decile of math FCAT achievement. As the coefficients on the quadratic and cubic FCAT terms are small, we only report the coefficient on the linear term in regression results.

${ }^{20}$ Many community colleges in Florida offer BA degrees; however, the nature of the data makes it difficult to distinguish credits earned at a community college in pursuit of a BA from credits earned at a community college in pursuit of a different degree. To ensure a clean measure of credits earned in pursuit of a BA (four-year credits), we drop the approximately 500 students who attempted a BA at a two-year institution and the 100 students who earned a BA at a two-year institution.

${ }^{21}$ Kane and Rouse (1998) report that a year of schooling at the two-year level is valued similarly in the labor market to one from a four-year school. But Acemoglu and Autor (2011) report a recent "convexification" of the returns to schooling in which each year of additional postsecondary schooling generates higher average value for all.

${ }^{22}$ The relatively large return to $\mathrm{CC}$ students with zero credits is puzzling. It might indicate some selection into these schools by unobservables not captured by our FCAT scores or other variables. In contrast, the near-zero return to those with no credits in BA programs suggests no such selection exists for these students.

${ }^{23}$ An exception to this literature is Clark and Martorell (2014) who find little evidence of sheepskin effects for high-school diplomas.

${ }^{24}$ When postsecondary GPA and math/science credits are added separately, the former generates a much larger decline in the value of the degree than the latter. Details are available from the authors. Also, the coefficients on math/science credits in 
Col. 4 cannot be directly compared to the returns to all credits since the latter is only for degree non-completers, while the former is for all students.

${ }^{25}$ See the Economic and Statistics Administration (2011) for recent evidence on the labor market values of STEM jobs, and Jacobson et al. (2005) for earlier evidence on the values of community college education with more technical courses and curricula, though for a sample of older displaced workers.

${ }^{26}$ Other attempts to control for selection (such as with high school GPA) generate similar results. Since FCAT has fewer missing observations, we include only FCAT scores.

${ }^{27}$ Johnson and Neal (1998) and Holzer and Dunlop (op. cit) show that racial gaps in earnings grow much smaller when we control for differences in education plus academic achievement. But the inclusion of quarters with zero earnings can strongly reduce the extent to which education and achievement account for the earnings gaps of black, relative to white, men.

${ }^{28}$ In estimated equations that contain no controls for high school achievement, our coefficients on having AA, BA, or graduate degrees in column 1 are .03, .04, or .07 higher, respectively, and the broad qualitative pattern of returns remains the same.

${ }^{29} \mathrm{We}$ find similar patterns across fields of study among those not completing degree programs, although the results are not quite as pronounced. Results are available from the authors.

${ }^{30} \mathrm{We}$ have estimated separate equations by race; findings are similar to the FRL results and are available from the authors.

${ }^{31}$ The National Governors' Association (2013) reports a widespread attempt to build "sectorial" training partnerships at the state and regional levels between community colleges and industry groups, though we have little data on the scale of student enrollments in programs generated through these partnerships. The National Council of State Legislatures (2014) reports that over half of all states are planning to at least partially use higher education academic outcomes to determine state subsidies to higher education institutions, and Holzer (2014) argues that employment outcomes should also be used to measure institutional performance as well. But the use of such outcomes without adjusting for the quality of student inputs could result in "cream-skimming" through higher admissions standards, among other potential unanticipated consequences.

${ }^{32}$ Long (2014) notes that remedial classes, to which many academically underperforming students are assigned in community colleges before they are allowed to take courses for credit, are generally ineffective and recommends a range of reforms. The College Board (2013) report on Pell grants also recommends a range of reforms designed to improve completion rates and subsequent earnings among low-income students.

${ }^{33}$ Models of high-quality career and technical education, such as apprenticeship, increasingly build the attainment of postsecondary credentials like AA degrees into their training model. See Lerman (2010).

Competing interests

The IZA Journal of Labor Policy is committed to the IZA Guiding Principles of Research Integrity. The authors declare that they have observed these principles. 
Institute for Research on Poverty, University of Wisconsin, July 2014; the CAPSEE conference, Washington DC, September 2014; and an anonymous referee for their discussion and comments. This research was supported by the CALDER postsecondary initiative, funded through grants provided by the Bill and Melinda Gates Foundation and the Smith Richardson Foundation to the American Institutes of Research.

Responsible editor: David Neumark

\section{Author details}

${ }^{1}$ American Institutes for Research (AIR)/CALDER, Washington, DC 20007, USA. ${ }^{2}$ Georgetown University and AIR/CALDER, Washington, DC 20007, USA. ${ }^{3}$ RTI International, Washington, DC, USA.

Received: 27 August 2014 Accepted: 29 November 2014

Published online: 23 January 2015

\section{References}

Acemoglu D, Autor D (2011) Skills, Tasks and Technologies: Implications for Employment and Earnings. In: Ashenfelter

O, Card D (eds) The Handbook of Labor Economics, vol 4, North Holland, Amsterdam

Altonji, J. Blom E, Meghir C 2012. "Heterogeneity in Human Capital Investments: High School Curriculum, College Major, and Careers," National Bureau of Economic Research Working Paper, Cambridge MA

Andersson F, Holzer H, Lane J (2005) Moving Up or Moving On: Who Advances in the Low-Wage Labor Market? Russell Sage Foundation, NewYork

Arcidiacono P, Aucejo E, Spenner K (2012) "What happens after enrollment? an analysis of the time path of racial differences in GPA and major choice," IZA J Labor Econ 1:Article 5

Arcidiacono P, Aucejo E, Hotz VJ (2013) "University Differences in the Graduation of Minorities in STEM Fields: Evidence from California", revise and resubmit American Economic Review

Bahr P, Dynarski S, Jacob B, Kreisman D (2014) "Labor Market Returns to Community College in Michigan." Unpublished manuscript, University of Michigan

Bailey M, Dynarski S (2011) Inequality in Postsecondary Education. In: Duncan G, Murnane R (eds) Whither Opportunity? Rising Inequality, Schools, and Children's Life Chances. Russell Sage Foundation, New York

Belfield C, Liu YT, Trimble MJ (2014) "The Medium-Term Labor Market Returns to Community College Awards: Evidence From North Carolina, New York. Center for Analysis of Postsecondary Education and Employment, NY

Borjas G (1987) Self-selection and the earnings of immigrants. Am Econ Rev 77(4):531-553

Bound J, Lovenheim M, Turner S (2009) Why Have College Completion Rates Declined? An Analysis of Changes in Student Preparation and College Resources. National Bureau of Economic Research Working Paper, Cambridge MA

Clark, D and P Martorell. 2014. "The Signaling Value of a High School Diploma" (with Paco Martorell). Journal of Political Economy. Forthcoming.

College Board. 2013. Rethinking Pell Grants. College Board, Washington DC

Economic and Statistics Administration (2011) STEM: Good Jobs Now and For the Future. Department of Commerce, US

Haskins R, Holzer H, Lerman R (2009) Promoting Economic Mobility by Increasing Postsecondary Education. The Pew Trusts, Washington DC

Holzer H (2014) Improving Employment Outcomes for Disadvantaged Students. Hamilton Project, The Brookings Institution, Washington DC

Holzer H, Dunlop E (2013) Just the Facts, Ma'am: Postsecondary Education and Labor Market Outcomes in the U.S. CALDER Working Paper, American Institutes for Research, Washington DC

Hoxby C, Turner S (2013) Informing Students About their College Prospects: A Proposal for Broadening the Expanding College Opportunity Project. The Hamilton Project, Brookings Institution, Washington DC

Institute of Education Sciences (2014) Digest of Education Statistics. U.S. Government Printing Office, Washington DC

Isaacs, J (2007). Economic Mobility of Families across Generations. Policy Brief, Center on Children and Families, the Brookings Institution, Washington DC

Jacobson L, Mokher C (2009) Pathways to Boosting the Earnings of Low-Income Students by Increasing their Educational Attainment. Hudson Institute, New York

Jacobson L et al (2005) The Impact of Community College on Older Workers: Should We Teach Old Dogs New Tricks?". Working Paper, Cornell ILR School

Jenkins D, Cho S-W (2012) Get with the Program: Accelerating Community College Entry Into and Completion of Programs of Study. Community College Research Center Working Paper, Teachers College, Columbia University

Jepsen C, Troske K, Coomes P (2014) The labor-market returns to community college degrees, diplomas, and certificates. J Labor Econ 32(1):95-121, University of Chicago Press

Johnson W, Neal D (1998) Basic Skills and the Black-White Earnings Gap. In: Jencks C, Phillips M (eds) The Black-White Test Score Gap. Brookings Institution, Washington DC

Kane T, Rouse C (1998) The labor market returns to Two-and four-year colleges. Am Econ Rev 85(2):600-614

Kreisman D, Dynarski S, Jacobs B, Bahr P (2013) The Returns to Couse Credits, Certificates and Degrees: Evidence from Michigan's Community Colleges. Unpublished, University of Michigan

Lerman R (2010) Expanding Apprenticeships: A Way To Improve Skills and Careers. The Urban Institute, Washington DC

Long BT (2014) Addressing the Academic Barriers to Success. In: Kearney M, Harris B (eds) Policies to Address Poverty in America. The Hamilton Project, Brookings Institution, Washington DC

Lovenheim M, Reynolds C (2011) Changes in postsecondary choices by ability and income: evidence from the national longitudinal surveys of youth. J Human Capital 5(1):70-109

Malamud O, Wozniak A (2008) The Impact of College Graduation on Geographic Mobility: Identifying Education Using Multiple Components of Vietnam Draft Risk, IZA Discussion Paper Series No. 3432

Mishel L, Roy J (2004) Rethinking High School Graduation Rates and Trends. Economic Policy Institute, Washington DC

Murnane R (2013) U.S. High school graduation rates: patterns and explanations. J Econ Lit 51(2):370-422 
National Center for Education Statistics (2013) Digest of Education Statistics. Institute of Education Sciences, Washington DC

National Council of State Legislatures. 2014. "Performance-Based Subsidies for Higher Education." http://www.ncsl.org/ research/education/performance-funding.aspx

National Governors' Association (2013) State Sector Strategies Coming of Age. Center for Best Practices, Washington DC Reardon S (2011) The Widening Academic Achievement Gap Between the Rich and the Poor: New Evidence and Possible Explanations.". In: Duncan G, Murnane R (eds) Whither Opportunity? Rising Inequality, Schools, and Children's Life Chances. Russell Sage Foundation, New York

Rosenbaum J, Deil-Amen R, Person A (2006) After Admission: From College Access to College Success. Russell Sage Foundation, New York

Scott-Clayton J (2011) The Shapeless River: Does a Lack of Structure Inhibit Students' Progress at Community Colleges?" Community College Research Center Working Paper, Teachers College, Columbia University

Swanson C (2004) Who Graduates? Who Doesn't? A Statistical Portrait of Public High School Graduation, Class of 2001. The Urban Institute, Washington, DC

Xu D, Jaggers S (2014) Examining Wage Trajectories of Community College Students Using a Growth Curve Modeling Approach

Submit your manuscript to a SpringerOpen ${ }^{\circ}$ journal and benefit from:

- Convenient online submission

- Rigorous peer review

- Immediate publication on acceptance

- Open access: articles freely available online

- High visibility within the field

- Retaining the copyright to your article

Submit your next manuscript at $>$ springeropen.com 